\title{
PSO-Based Adaptive Hierarchical Interval Type-2 Fuzzy Knowledge Representation System (PSO-AHIT2FKRS) for Travel Route Guidance
}

\author{
Mariam Zouari ${ }^{\circledR}$, Student Member, IEEE, Nesrine Baklouti ${ }^{\circledR}$, Senior Member, IEEE, \\ Javier Sanchez-Medina ${ }^{\circledR}$, Senior Member, IEEE, Habib M. Kammoun ${ }^{\circledR}$, Senior Member, IEEE, \\ Mounir Ben Ayed ${ }^{\circledR}$, Senior Member, IEEE, and Adel M. Alimi ${ }^{\circledR}$, Senior Member, IEEE
}

\begin{abstract}
Urban Traffic Networks are characterized by their high dynamics and increased traffic congestion cases, leading to a more complex road traffic management. The present research work suggests an innovative advanced vehicle guidance system based on Hierarchical Interval Type-2 Fuzzy Logic model optimized by the Particle Swarm Optimization (PSO) method. Indeed, this system allows an intelligent and prompt adjustment of the road traffic network in a dynamic way and improves the entire road network quality, particularly in case of congestions or jams, considering real-time traffic information. The best followed road is selected according to the quality of traffic and route length, together with contextual factors pertaining to the driver, the environment, and the path. The proposed system is executed and simulated using SUMO (Simulation of Urban Mobility), for which four large areas situated in the cities of Sfax, Luxembourg, Bologna and Cologne have been tested. The simulation results proved the effectiveness of learning the Hierarchical Interval Type-2 Fuzzy Logic model using PSO real time technique to accomplish multi-objective optimality regarding two criteria: number of cars that attain their destination and average travel time. The obtained results have confirmed the efficiency of the proposed system.
\end{abstract}

Index Terms-Hierarchical interval type-2 fuzzy system, traffic congestion, dynamic travel route guidance, traffic simulation, knowledge representation, particle swarm optimization.

\section{INTRODUCTION}

D YNAMIC Traffic Management is a very important component of human life and economy that leads to positive effects on throughput, pollution, and safety [1]. The objective of road traffic management is not only to improve traffic fluency on road networks, assigning dynamically the traffic flows, but also to decrease the number of traffic

Manuscript received July 19, 2019; revised February 2, 2020 and June 11, 2020; accepted July 30, 2020. Date of publication August 31, 2020; date of current version February 2, 2022. This work was supported by the Ministry of Higher Education and Scientific Research of Tunisia under Grant LR11ES48. The Associate Editor for this article was P. Wang. (Corresponding author: Mariam Zouari.)

Mariam Zouari, Nesrine Baklouti, Habib M. Kammoun, Mounir Ben Ayed, and Adel M. Alimi are with the Research Groups in Intelligent Machines (REGIM-Lab), National Engineering School of Sfax (ENIS), University of Sfax, Sfax 3038, Tunisia (e-mail: mariam.zouari@ieee.org; nesrine.baklouti@ieee.org; habib.kammoun@ ieee.org; mounir.benayed@ieee.org; adel.alimi@ieee.org).

Javier Sanchez-Medina is with the Innovation Center for the Information Society (CICEI), University of Las Palmas de Gran Canaria, 35017 Las Palmas de Gran Canaria, Spain (e-mail: javier.sanchez.medina@ieee.org).

Digital Object Identifier 10.1109/TITS.2020.3016054 congestions states and their negative effects (i.e. waiting time, drivers stress, delays, carbon footprint or emergency vehicles blocking) [2], [3].

To disperse traffic congestions in urban networks, the re-searchers mainly interested in traffic assignment and signal optimization models, which may be unable to afford accurate and fast route guidance due to the absence of real-time information and individual traffic demands. The dynamic route guidance system can provide real-time information and optimal driving paths to destination for the driver, considering current and anticipated traffic conditions [4]-[7]. Thus, there is a need for a predictive system that offers each driver in real time, the best choice at each intersection for every destination and present traffic state pair. However, conventional traffic management decisions are made driven simply by engineers' experience and static and outdated traffic behavior assumptions, with the subsequent gap to an utmost efficient use of the traffic infrastructures. Moreover, the measurement of several decisions, objectives and constraints are commonly difficult to be realized by crisp values. Hard computing models cannot be treated effectively with the transport decision-makers ambiguities and uncertainties. To deal with these problems in road perceptions, the application of Interval Type-2 Fuzzy Logic is considered an appropriate mathematical device for decision making in transportation engineering [8]. Actually, guaranteeing control by using Interval Type-2 Fuzzy Logic has been proven to be a great success in a large variety of applications [9]-[12]. Nevertheless, the performance of a designed controller is likely to change depending on the method used in the design process. The definition of the appropriate parameters of membership functions values of a Fuzzy Logic System (FLS) in both antecedent and ensuing parts is difficult because their calculation is time-consuming. The major problem in designing an FLS lies in how to fix the suitable fitting membership values.

In most previous recent works, attention has extensively been drawn to the optimization of fuzzy systems. Indeed, optimization algorithms can be a useful tool as they can solve nonlinear problems, well-constrained or even NP-hard problems. Among the most used optimization methods, we can find: Genetic Algorithms (GA) [13], [14], Ant Colony Optimization algorithms (ACO) [15] and PSO [16], etc. 
PSO is a new evolutionary optimization technique that is quick, simple, and likely to be used for looking for the best solution in a wide search space. In fact, PSO has been proven to be effective in several applications [17]-[19].

This paper describes the application of PSO as optimization methods to instantly tune the MFs of our HIT2FKRS model and design optimal Fuzzy Logic Controllers (FLC) which assist the driver to achieve his/her destination, while eluding congestions. The purpose of the suggested system is to adjust promptly the road traffic in a dynamic way and ameliorate the entire road network quality, particularly in case of congestions or jams, considering real-time traffic information and drivers' travel time to attain their destinations. Thanks to our advanced system, the user-optimal route is selected before going through each intersection according to the quality of traffic and route length, together with contextual factors pertaining to the driver, the environment, and the path. Essentially, it pertains to the automatic diversion of the traffic into the ideal choice of a set of alternatives or the combination of alternatives towards each vehicle's destination node. In our daily life, our system can be widely used as a useful mobile application to facilitate driving in familiar or unfamiliar environment providing drivers with the best route choice. To corroborate our methodology, we have used traffic simulation. The open source microscopic road traffic simulator (SUMO) has been used [20].

Concretely, our contributions are summarized as follows:

- We propose a PSO based Adaptive Hierarchical Interval Type-2 Fuzzy Knowledge Representation System (PSOAHIT2FKRS) for travel route guidance. The suggested system is carried out to select the best followed road to achieve destination, according to the quality of road traffic in real time, evading crowded areas, and thus conceding route length and time.

- We develop a HIT2FLS for itinerary evaluation, combined six contextual factors associated with the decision-making process, namely environmental conditions, path and driver characteristics.

- Our proposal is tested under different conditions of real road network dimension and traffic density.

- Compared with Dijkstra method, HIT2FKRS and HIT1FKRS, our suggested system (PSO-AHIT2FKRS) provides multiobjective optimality regarding two criteria: number of cars that attain their destination and average travel time.

The rest of the paper is organized as follows. Section 2 overviews the use of Fuzzy Logic, Interval Type-2 Fuzzy Logic and PSO for the route choice problem. Section 3 presents the basic concepts of Particle Swarm Optimization and the theory of Interval Type-2 Fuzzy Logic. The details of the proposed Evolutionary Hierarchical Interval Type-2 Fuzzy Knowledge Representation System for travel route guidance are displayed in the fourth section. Indeed, this section firstly describes the PSO base Adaptive Hierarchical Interval Type-2- Fuzzy Logic System (PSOAHIT2FLS) for route choice evaluation and its components, and secondly presents the significant role of the a recognized traffic simulator SUMO. Section 5 exhibits the conducted simulations using SUMO, as well as the analysis of the results obtained to validate our approach. The paper ends with a conclusion and some perspectives for future research work.

\section{Literature REVIEW OF ROAD TRAFFIC MANAGEMENT}

Thanks to the effective techniques and methodologies from artificial intelligence which are able intelligently to solve the complex problems related to transportation systems [21], researchers have been interested in their use. We would like to give a succinct survey of few significant research works in road traffic management which aim not only to manage traffic but also to optimize it. For instance, Fu [22] has proposed an adaptive routing algorithm for an in-vehicle navigation system with real-time information. The variable of the link travel time was taken randomly with known mean and standard deviation. The realization of travel time can be estimated based on real-time data collected over the links. While, in [23], a route choice model, using a hybrid probabilistic-possibilistic model has been proposed. This model is used to quantify the latent attractiveness of alternative routes. However, in this research work, the prediction was poor in case the driver's behavior was heterogeneous. In another work [24], Wang et al. have introduced real-time route guidance in large-scale express ring roads focusing on feedback routing performance, in case of incidents. Real-time dynamic user equilibrium (DUE) was established within the freeway network by suggesting alternative routes to users. Ding et al. [25] have investigated a real-time vehicle route guidance system based on V2V (Vehicle to Vehicle) and V2R (Vehicle to Roadside) communication. This work has mainly focused on two parts which are: a smart route query and reply strategy of route finding algorithm, as well as a detour algorithm in order to bypass void areas without running cars. Literature in road traffic management is quite extensive, thus our focus will be on the review of the latest models that rely on Fuzzy Logic, Interval Type-2 Fuzzy Logic and PSO.

\section{A. Fuzzy Logic-Based Road Traffic Management}

Many classical methods have been suggested to primarily contend with the problem of choosing the route using the discrete choice models such as Logit and Probit models, in the majority of cases [26], [27]. Such techniques cannot adapt with the ambiguities and uncertainties of the perceptions of environment, and thus they are considered as an inefficiency gap to account for the dynamicity and complexity of transportation systems. Hence, many researchers are resolving transportation problems by using soft computing, specifically Fuzzy Logic, as a useful tool for treating those uncertainties [28]. Among the well-known transportation related engineering problems traffic light management, route guidance, traffic assignment and road traffic management [2], [6], [29]-[31] can be mentioned.

Table I presents some research works pertaining to Fuzzy Logic-based road traffic management. Despite the fact that Type-1 Fuzzy Logic System (T1FLS) is considered as one of the well-known types of FLS, it remains an unsatisfactory representation of the real-time traffic uncertainties. Furthermore, 
TABLE I

Relevant Related Works on Type-1 Fuzzy Logic- Based Road Traffic MANAgement

\begin{tabular}{|c|c|c|c|c|c|}
\hline Refs & Year & Objective & Main features & Strength & Weakness \\
\hline$[2]$ & 2014 & $\begin{array}{l}\text { Road Traffic } \\
\text { management }\end{array}$ & $\begin{array}{l}\text { - Hierarchical fuzzy model } \\
\text { - Multi-agent system based } \\
\text { on the ant colony behavior }\end{array}$ & $\begin{array}{l}\text { - Fine-tune the road traffic in } \\
\text { the network in line with the real } \\
\text { times using an adaptive vehicle } \\
\text { route guidance system }\end{array}$ & $\begin{array}{l}\text { - No real time management } \\
\text { - No real networks }\end{array}$ \\
\hline [6] & 2014 & $\begin{array}{l}\text { Road Traffic } \\
\text { management }\end{array}$ & $\begin{array}{l}\text { - Analytical hierarchy process } \\
\text { using a Fuzzy Logic } \\
\text { (AHP-FUZZY) }\end{array}$ & $\begin{array}{l}\text { - Reasonable optimal route choice } \\
\text { combining the road segments' cost } \\
\text { and the overall O/D cost. } \\
\text { - Decrease Traffic congestion. }\end{array}$ & $\begin{array}{l}\text { - Restricted number of route } \\
\text { choice criteria }\end{array}$ \\
\hline [29] & 2014 & $\begin{array}{l}\text { Traffic congestion } \\
\text { prediction system }\end{array}$ & $\begin{array}{l}\text { - Hierarchical fuzzy system } \\
\text { - Genetic algorithm }\end{array}$ & $\begin{array}{l}\text { - Decreases the input variables' } \\
\text { size while preserving } \\
\text { a good precision }\end{array}$ & $\begin{array}{l}\text {-No simulations } \\
\text {-Test the study on only } \\
\text { segment of highway }\end{array}$ \\
\hline$[30]$ & 2016 & $\begin{array}{l}\text { Congested road } \\
\text { notification system }\end{array}$ & $\begin{array}{l}\text { - Fuzzy Logic inVANET } \\
\text { context aware congested road }\end{array}$ & $\begin{array}{l}\text { - Best assessment of the current } \\
\text { situation of vehicle compared } \\
\text { to the congestion situation. }\end{array}$ & - Low interpretability of results \\
\hline [31] & 2017 & $\begin{array}{l}\text { Cyclists route } \\
\text { guidance system }\end{array}$ & - Fuzzy Inference System & $\begin{array}{l}\text { - Reasonable optimal route choice } \\
\text { system to bike users which } \\
\text { is embedded in a mobile platform. }\end{array}$ & $\begin{array}{l}\text { - No real networks } \\
\text { - Restricted number of route } \\
\text { choice criteria }\end{array}$ \\
\hline
\end{tabular}

TABLE II

Relevant Related Works on Type-2 Fuzzy Logic-Based Road TrafFic Management

\begin{tabular}{|c|c|c|c|c|c|}
\hline Refs & Year & Objective & Main features & Strength & Weakness \\
\hline [8] & 2011 & $\begin{array}{l}\text { Urban traffic manage- } \\
\text { ment }\end{array}$ & $\begin{array}{l}\text { - Multi-agent signal control } \\
\text { - Type-2 Fuzzy decision module }\end{array}$ & $\begin{array}{l}\text { - Optimize the green time } \\
\text { - Diminish vehicles total delay } \\
\text { - Diminish vehicles' total travel time }\end{array}$ & $\begin{array}{l}\text { - No vehicle route } \\
\text { guidance }\end{array}$ \\
\hline [34] & 2012 & Traffic signal control & $\begin{array}{l}\text { - Distributed multi-agent } \\
\text { architecture } \\
\text { - Type-2 Fuzzy decision system }\end{array}$ & $\begin{array}{l}\text { - Determine the green time that } \\
\text { diminishes the overall delay. }\end{array}$ & $\begin{array}{l}\text { - No results for } \\
\text { real networks }\end{array}$ \\
\hline [35] & 2014 & Traffic congestion & $\begin{array}{l}\text { - Type-2 Fuzzy Logic } \\
\text { - Genetic algorithm }\end{array}$ & $\begin{array}{l}\text { - Decrease the waiting line length } \\
\text { - Diminish vehicles delay at the } \\
\text { intersection. }\end{array}$ & $\begin{array}{l}\text { - No results for } \\
\text { real networks }\end{array}$ \\
\hline [36] & 2016 & $\begin{array}{l}\text { Traffic flow data fore- } \\
\text { casting }\end{array}$ & - Interval Type-2 Fuzzy sets theory & $\begin{array}{l}\text { - Lessen the impact of noise } \\
\text { from the detection data. }\end{array}$ & $\begin{array}{l}\text { - One road } \\
\text { network test }\end{array}$ \\
\hline [37] & 2017 & $\begin{array}{l}\text { Road traffic manage- } \\
\text { ment }\end{array}$ & - Interval Type-2 Fuzzy sets theory & $\begin{array}{l}\text { - Reduction of the travel time } \\
\text { and congestion situations } \\
\text { - Improvement of traffic quality. }\end{array}$ & $\begin{array}{l}\text { - One road } \\
\text { network test }\end{array}$ \\
\hline [38] & 2018 & Traffic Signal Control & $\begin{array}{l}\text { - Interval Type-2 Fuzzy sets theory } \\
\text { - Gravitational search algorithm }\end{array}$ & $\begin{array}{l}\text { - Adjusting the green time based } \\
\text { on the number of vehicles from the } \\
\text { intersection to the downstream } \\
\text { intersection } \\
\text { - Extend green wave band }\end{array}$ & $\begin{array}{l}\text { - Arterial traffic } \\
\text { that includes five } \\
\text { intersections }\end{array}$ \\
\hline
\end{tabular}

road traffic management problems often face several sources and elevated levels of uncertainty [32]. Therefore, it is crucial to integrate Type-2 Fuzzy Logic in road traffic management.

\section{B. Type- 2 Fuzzy Logic-Based Road Traffic Management}

Type-2 Fuzzy set [33] represents an extension of the concept of Type-1-Fuzzy set. Indeed, Type-2 Fuzzy sets offer a more precise way to design uncertainty in a system. For instance, they can handle linguistic uncertainties by modeling information's vagueness and lack of reliability.

Numerous prominent research works [8], [34]-[38], summarized in Table II, have dealt with many problems in traffic management that have been solved applying IT2FLS. Most of the developed models for route selection depended on few possible alternatives taking into account the restricted number of criteria to prevail over the rule-explosion problem. This is an enhancement for the present work to further continue and rely on a Hierarchical Interval Type-2 Fuzzy Logic system for the itinerary evaluation that can take into consideration numerous selection criteria to select the best itinerary.

\section{Swarm Intelligence (PSO)-Based Road Traffic} Management

The extensive use of swarm intelligence was to model complex traffic and transportation processes [39]. In fact, Ant Colony Optimization (ACO) has been extensively used to solve transportation problems, such as Vehicle Routing Problem (VRP) and Traveling Salesman Problem (TSP) [40], [41]. However, there exist a few publications based on swarm intelligence, especially PSO, to solve road traffic management problems. PSO algorithm is a population intelligence algorithm that has good performance in optimization. The most important features of the pertinent related works on traffic management based on PSO are summarized in Table III.

In most of the presented studies, the PSO was applied to control and optimize traffic signals. Moreover, some of these studies are not based on real scenarios and the dynamic real-time information during the journey. This is the reason behind taking the initiative to test the reliability of PSO for learning our HIT2FLS to dynamic travel route guidance using real scenarios. 
TABLE III

RELEVAnt Related Works on SWARm InTELligence (PSO)-BASEd RoAd TrafFic MANAGEMEnT

\begin{tabular}{|c|c|c|c|c|c|}
\hline Refs & Year & Objective & Main features & Strength & Weakness \\
\hline [17] & 2012 & $\begin{array}{l}\text { Traffic } \\
\text { light control }\end{array}$ & $\begin{array}{l}\text { - PSO- based } \\
\text { fuzzy controller }\end{array}$ & $\begin{array}{l}\text { - Enhance the traffic efficiency } \\
\text { of new roundabouts. }\end{array}$ & $\begin{array}{l}\text { - Only roundabout for } \\
\text { simulation }\end{array}$ \\
\hline [42] & 2013 & $\begin{array}{l}\text { Traffic } \\
\text { light control }\end{array}$ & $\begin{array}{l}\text { - PSO for finding efficient } \\
\text { traffic signal cycle programs } \\
\text { - SUMO }\end{array}$ & $\begin{array}{l}\text { - Quantitative enhancements for } \\
\text { the both objectives: vehicles' number } \\
\text { that attain their destination and overall } \\
\text { travel time. }\end{array}$ & $\begin{array}{l}\text { - One of scenarios is } \\
\text { virtual. }\end{array}$ \\
\hline [43] & 2013 & $\begin{array}{l}\text { Traffic } \\
\text { Signal Control }\end{array}$ & $\begin{array}{l}\text { - Bayesian Network model coupled with } \\
\text { the Cellular Automaton model for building- } \\
\text { up a probability model for traffic } \\
\text { jam. } \\
\text { - PSO based on the probability model }\end{array}$ & $\begin{array}{l}\text { - Obtain optimal traffic signals } \\
\text { - Minimize the probability of traffic } \\
\text { jam }\end{array}$ & $\begin{array}{l}\text { - No real traffic data. } \\
\text { - Simple road network }\end{array}$ \\
\hline [44] & 2016 & $\begin{array}{l}\text { Traffic } \\
\text { Signal Control }\end{array}$ & $\begin{array}{l}\text { - Cell Transmission Model (CTM) } \\
\text { to model the online traffic network } \\
\text { - PSO for optimizing the control of the } \\
\text { traffic signal network }\end{array}$ & $\begin{array}{l}\text { - Minimize the overall delay on the } \\
\text { traffic network } \\
\text { - Lower the fuel consumption. }\end{array}$ & $\begin{array}{l}\text { - No real traffic data. } \\
\text { - Sub-network to test }\end{array}$ \\
\hline [45] & 2017 & $\begin{array}{l}\text { Traffic } \\
\text { management } \\
\text { and traffic light } \\
\text { control }\end{array}$ & $\begin{array}{l}\text { - Novel traffic management model } \\
\text { based on Multi-Objective Particle } \\
\text { Swarm Optimization method } \\
\text { - SUMO }\end{array}$ & $\begin{array}{l}\text { - Optimize vehicle re-routing } \\
\text { - Optimize signal timing. }\end{array}$ & $\begin{array}{l}\text { - Virtual road network. } \\
\text { - Nine-node network for } \\
\text { test }\end{array}$ \\
\hline [46] & 2019 & $\begin{array}{l}\text { Traffic flow } \\
\text { guidance }\end{array}$ & $\begin{array}{l}\text { - A multi-objective optimization model } \\
\text { of traffic flow guidance based on } \\
\text { PSO method }\end{array}$ & $\begin{array}{l}\text { - Enhance the traffic efficiency, } \\
\text { - Decrease the travel time and delay } \\
\text { - Reduce the traffic jam. }\end{array}$ & $\begin{array}{l}\text { - Limited number of } \\
\text { intersections } \\
\text { - Simple road network }\end{array}$ \\
\hline
\end{tabular}

\section{Synthesis}

To obtain an effective road traffic management, the traffic models necessitate maximum real-time information. However, most of the developed models, detailed in the previous subsections, are used to evaluate only few possible alternatives taking into account a restricted number of criteria to select the best route to follow. The selection of the optimal route with limited criteria such as distance and travel time is not always efficient. Moreover, the majority of research works have not dealt with models close to reality i.e. by considering dynamic information in large and real networks. To overcome these limitations, this paper proposes a route guidance model based on adaptive Hierarchical Interval Type-2 Fuzzy Logic that can take into consideration the most important contextual factors which assist drivers to take decision for choosing the best itinerary. In addition, to achieve the desired level of robust performance for controllers, the PSO algorithm was performed for evolving the proposed hierarchical model controller. For evaluating our system, four big realistic traffic scenarios have been tested using the simulator SUMO. Actually, the proposed system contributes to the improvement in traffic fluency while taking into account the real-time model road traffic information.

\section{Fundamentals of PARTICLE SWARM Optimization AND INTERVAL TYPE-2 FUZZY LOGIC}

This section introduces the basic concepts of PSO and theory of the Interval Type-2 Fuzzy Logic which is a generation of Type-1 Fuzzy Logic.

\section{A. Particle Swarm Optimization (PSO)}

PSO is a simple and robust technique of optimization proposed by Eberhart and Kennedy [16]. It was inspired by the social behavior of birds within a flock. It is an evolutionary technique that looks like Genetic Algorithms (GA). PSO operates with a solution population called particles and the entire population is called the swarm. The swarm is initialized with random solutions. A swarm is composed of $\mathrm{N}$ particles in an $\mathrm{M}$ dimensional search space. The values of position and velocity are denoted for each particle of the swarm which are in range $\left[X_{\min }, X_{\max }\right]$ and $\left[V_{\min }, V_{\max }\right]$, respectively. Each particle consecutively adjust its position on the basis of its own experience and to the experience of neighboring particles, using the best position encountered by itself and its neighbors. All particles of the swarm have objective values that are assessed by a fitness function, to be optimized.

In what follows the equations representing the velocity and the position of a $j^{\text {th }}$ particle respectively:

$$
\begin{aligned}
V_{j}(t t+1)= & w * V_{j}(t t)+C_{1} * r_{1} *\left(p_{\text {best }}-X_{j}(t t)\right) \\
& +C_{2} * r_{2} *\left(g_{\text {best }}-X_{j}(t t)\right) \\
X_{j}(t t+1)= & X_{j}(t t)+(1-w) V_{j}(t t+1)
\end{aligned}
$$

where $C_{1}$ and $C_{2}$ are constant factors, $w$ is the inertia weight. $r_{1}$ and $r_{2}$ are random numbers in $[0,1]$. p pest denotes the particle's best position. $g_{\text {best }}$ is the global best position of the swarm. The steps of PSO algorithm are explained as follows:

- Step1: Swarm initialization: the swarm size, location and velocity of each particle and the random parameters.

- Step2: Calculation of the objective value of individual particle. Assessment of the best position of the particle and the global best position of the swarm. Then, the particle updates its velocity and position according to equations (1) and (2).

- Step3: Termination on some stopping conditions or return to step 2.

- Step4: Finding the global optimal solution which offers the latest gbest.

The PSO algorithm is used in several engineering problems [47]-[52] indicating a successful performance, even in comparison with other contemporary optimization techniques [53]. Despite that, the use of PSO is still limited for 


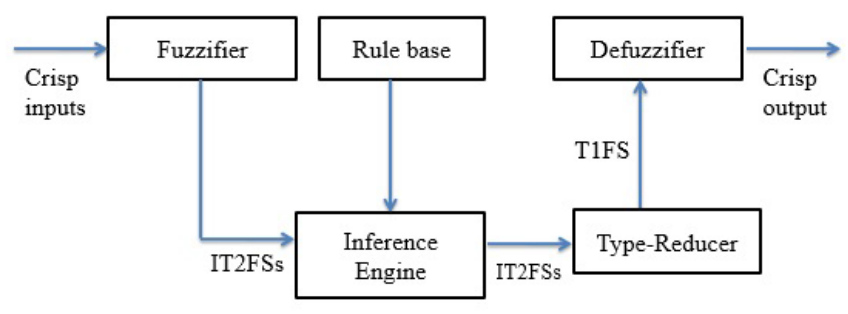

Fig. 1. The structure of a Type-2-FLS.

travel route guidance and other problems related to the road traffic management.

\section{B. Interval Type-2 Fuzzy Logic}

Interval Type-2 Fuzzy Sets (IT2FSs), originally introduced by Zadeh [54], provide additional degrees of freedom in Mamdani and TSK Fuzzy Logic Systems (FLSs), which can be efficient in situations where lots of uncertainties and imprecision are present [55]. The Interval Type-2 Fuzzy Logic Systems (IT2FLS) have the potential to afford better performance than a T1FLS. As a result of the uncertainties present in the surrounding environments, T1FLS might not be suitable because they may cause deterioration in the FLS performance. We might stop wasting time in frequently tuning or redesigning the T1FLS so that it can deal with the various encountered uncertainties. IT2FLSs employing IT2FSs offer possibility to handle elevated levels of uncertainties to provide good performances and better modeling. An Interval Type2 Fuzzy set is characterized by a fuzzy membership function, so the membership value for each element of this set is a fuzzy set in $[0,1]$, unlike a Type-1 Fuzzy set in which the membership value is a crisp number in $[0,1]$.

The membership function (MF) of IT2FLS is called the footprint of uncertainty (FOU) which is bounded up by a lower membership function (LMF) and by an upper membership function (UMF). The FOU of IT2FSs provides an extra dimension which gives additional degrees of freedom to model and handle uncertainties [56]. An IT2FLS is very similar in structure to T1FLS. The only difference is the extra output process component which is called the type-reducer before defuzzification, as can be seen in Figure 1 for a Mamdani model [57].

In details, an IT2FLS works as follows: the crisp inputs are first fuzzified to input Type-2 Fuzzy sets that are fed to the inference engine which maps the input Type-2 Fuzzy sets to output Type-2 Fuzzy sets using the rule base. The output set is then processed by the type-reducer in the type reduction section that generates a Type- 1 output set. An IT2FS, named $\widetilde{A}$, is expressed in the universe of discourse $\mathrm{X}$ by a Type2 membership function $\mu_{\widetilde{A}}(x, u)$ which is equal to 1 :

$$
\widetilde{A}=\int_{x \in X} \int_{u \in J} 1 /(x, u) J_{x} \in[0,1]
$$

$L M F$ and $U M F$ are expressed by $\underline{\mu}_{\widetilde{A}}(x)$ and $\bar{\mu}_{\widetilde{A}}(x)$ respectively. So, the $F O U$ is defined by the following relation:

$$
\operatorname{FOU}(\widetilde{A})=\bigcup_{x \in X} u \in J_{x} ; J_{x}=\left[\bar{\mu}_{\widetilde{A}}(x), \underline{\mu}_{\widetilde{A}}(x)\right]
$$

\section{Adaptive Fuzzy Logic Control By PSO OPTIMIZATION}

To achieve the desired level of robust performance for a controller, the exact tuning of the membership functions is very important. This paper intends to apply PSO algorithm to dynamically adjust the MFs of our HIT2FKRS model and design optimal Fuzzy Logic Controllers (FLC). The suggested PSO algorithm consist of the following sequential steps:

- Step1: Set the number of fuzzy sets $\mathrm{N}$ for each fuzzy variable and the initial fuzzy rules. Define the initial individuals of PSO for the control parameters of Fuzzy Logic Controller (FLC). All parameters of every FLC are tuned through the optimization algorithm. Every FLC is assessed through the objective function. Fetch the best fitness value (pbest).

- Step2: Update the parameter value of FLC whilst controlling the maximum velocity of particle.

- Step3: After the update of velocities, every particle modifies its position by the following equations:

$$
\begin{aligned}
v_{i m f}(t t+1)= & w \times v_{i m f}(t t)+c 1 \times r 1() \\
& \times\left(\text { pbest }_{\text {imf }}(t t)-x_{i m f}(t t)\right)+c 2 \times r 2() \\
& \times\left(G(t t)-x_{i m f}(t t)\right) \\
x_{i m f}(t t+1)= & x_{i m f}(t t)+v_{i m f}(t t+1)
\end{aligned}
$$

where $v_{i m f}$ is called the velocity of particle $\mathrm{i} ; x_{i m f}$ represents the position of particle $i$ with objective value fitness; $t t$ is the time step; $w$ indicates the inertia weight; $r 1()$ and $r 2()$ are random values in the range [0,1]; $c 1, c 2$ are acceleration parameters; pbestimf denotes the local best of particle $i ; G$ indicates the global best in the swarm.

- Step4: Readjust the local best pbest and the global best G.

- Step5: Termination on some stopping conditions or return to step 2.

- Step6: The latest global best G generated from the FLC represents the optimal FLC.

At each step time, the PSO algorithm trains the parameters of FLC to instantly extract the best values which assist the driver to achieve his/her destination, while eluding congestions.

In the process of fuzzy membership function adjusting via PSO, each particle is shaped to represent the fuzzy control rules and their corresponding MFs parameters of the FLC's inputs and outputs. Each particle represents a potential solution. These parameters are used to define the particles of PSO algorithm and look for the global best fitness.

\section{Architecture of Our PSO Based Adaptive HIERARCHICAL INTERVAL TYPE-2 FUZZY KNOWLEDGE REPRESENTATION SYSTEM (AHIT2FKRS)}

This section presents in details the suggested PSO based Adaptive Hierarchical Interval Type-2 Fuzzy Knowledge Representation System (PSO based AHIT2FKRS) for travel route guidance. The objective of the suggested system is the selection of the best route for every car according to the quality of road traffic in real time by the addition of other contextual factors associated with the decision-making process, 


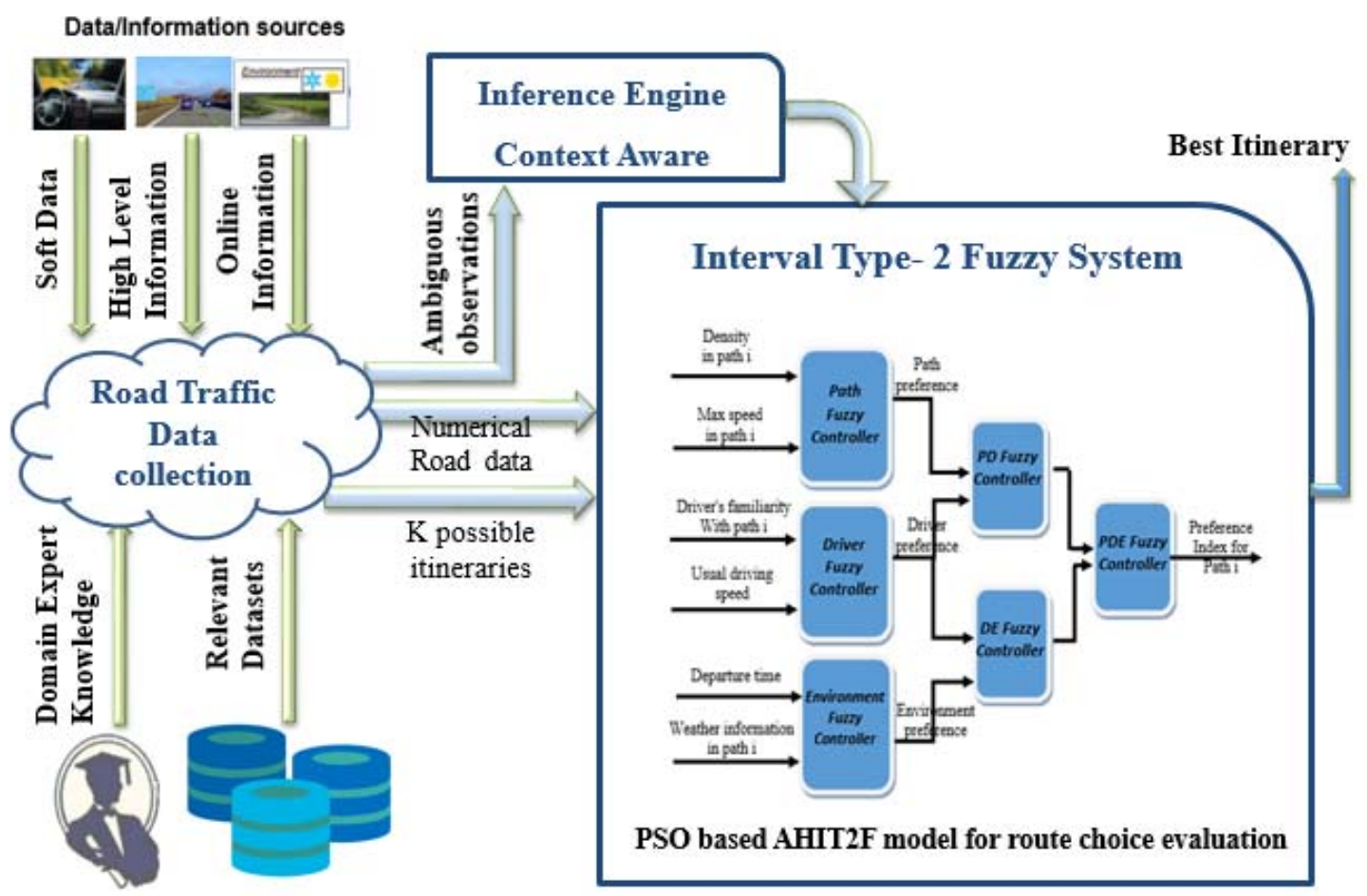

Fig. 2. The architecture of the suggested system.

namely environmental conditions, path and driver characteristics. Thus, the suggested PSO based AHIT2FKRS for travel route guidance draws in two main phases. Concerning the first one, it is initiated from a PSO based AHIT2FL model us to choose the best route among the possible routes by offering information from the simulator SUMO, which has an influence on the route choice. As for the second phase, it hinges on the road traffic simulator SUMO.

\section{A. PSO Based Adaptive Hierarchical Interval Type-2 Fuzzy (AHIT2F) Model for Travel Route Guidance}

To reach destination, the proposed system increases the vehicles' average speed on the road network, whilst choosing the best route in terms of the road traffic quality in real-time. It is noteworthy to note that the best itinerary is selected according to the distance and the expected travel time, together with contextual factors linked to the driver, the environment and path. Because of the uncertainty, ambiguity and dynamicity of these factors, it is very difficult to formulate a suitable deterministic mathematical model. However, the development of Interval Type-2 Fuzzy Logic seems justified in this situation thanks to its ability to approximate a real continuous function with a good accuracy. With respect to the increasingly numerous choice criteria for selecting the best alternative, the use of Fuzzy Logic for itinerary choice problem including a big number of inputs causes the rule-explosion problem. The use of hierarchical architecture seems to be the good solution to surmount this problem. It means that the number of rules increases linearly related to the number of inputs rather than exponentially. In fact, the Standard Fuzzy System (SFS) with large input dimensions is decomposed into several lower dimensional sub fuzzy systems which are related in a hierarchical way. For example, suppose that we have a SFS which has six inputs each represented by three fuzzy sets, in this case the number of rules is equal to $3^{6}=729$ rules. Nevertheless, in the case of hierarchical fuzzy system like it is shown in Figure 2, each SFS has $3^{2}$ rules, as a result, the total number of rule is equal to $6 * 3^{2}=54$ rules.

This indicates that the system enjoys a significant decrease in the total number of rules due to its hierarchical structure. As regards the traffic management, this tool was used effectively in some research works [2], [58] and [59]. The present work develops an HIT2FLS for itinerary evaluation, fed with six fuzzy inputs influencing itinerary selection decision which are density, maximum speed allowed in the path, familiarity of the driver with the path i, usual driving speed, departure time and weather information. The structure of HIT2FLS for itinerary evaluation is presented in Figure 2. In some fuzzy hierarchical architectures, outputs can be the inputs of the following fuzzy layer [58], compromising the interpretability of the overall model since the intermediate outputs do not possess a physical meaning.

To overcome this problem, the combination of inputs was considered as a resort to reduce limitations linked to the physical meaning loss in intermediate outputs/inputs. Indeed, inputs are reorganized in three categories, namely the route criteria, driver criteria and environment criteria.

Our HIT2FLS has six fuzzy controllers, each of which has two inputs and one output. The fuzzy sets of each input are presented in Figure 3. The first Path Fuzzy Controller input is the density of path $i$ which has three membership functions, 'free flow', 'synchronized flow' and 'Jam 

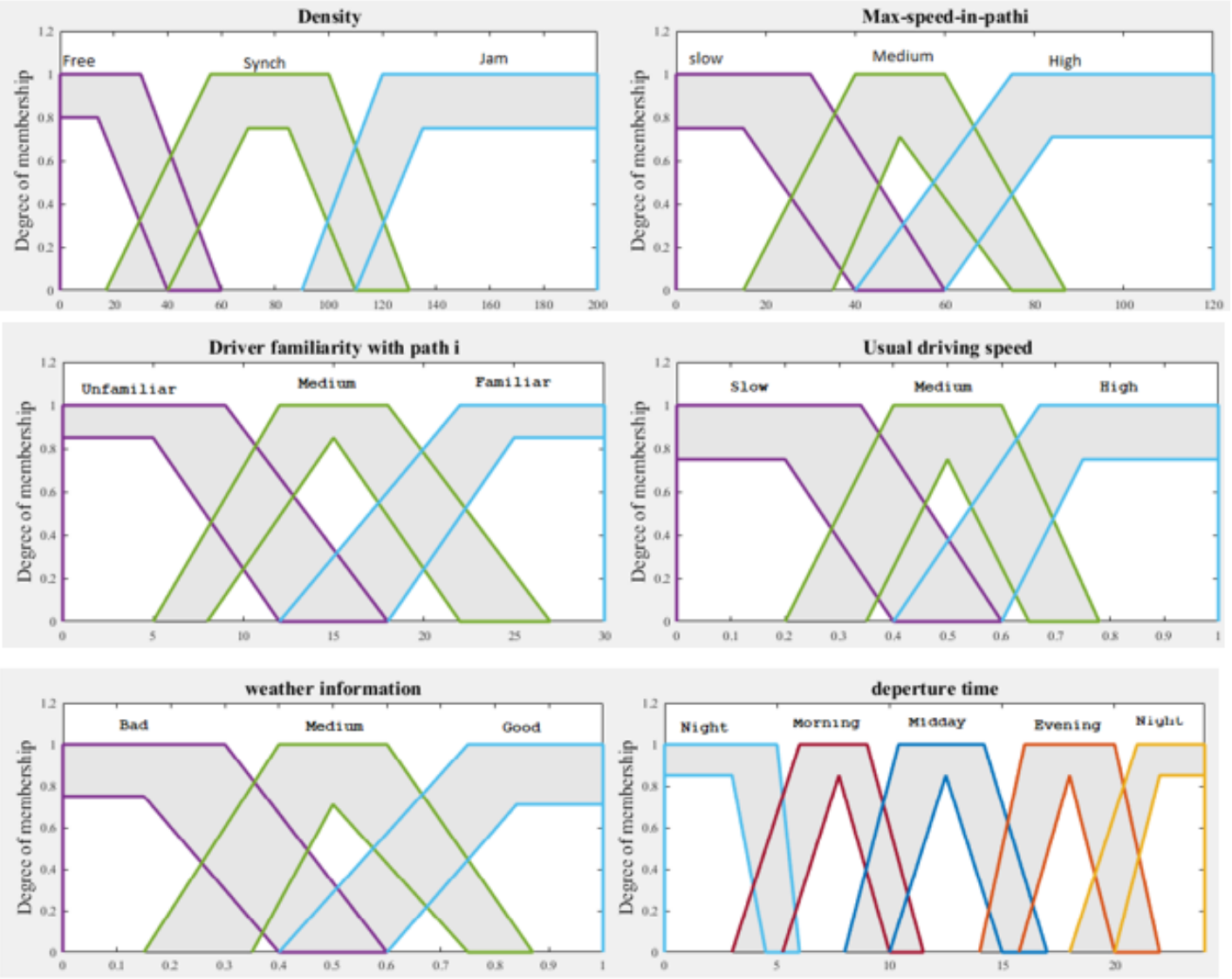

Fig. 3. Inputs membership functions.

flow'. The second input is the maximum speed in path $i$ which is represented also by three membership functions corresponding to 'slow', 'medium' and 'high'. Whereas the Path Fuzzy Controller output is the path preference, it has three membership functions which are 'weak', 'medium' and 'strong'. The inputs of Driver Fuzzy Controller are driver's familiarity with path $i$ and driving speed of the driver. The driver's familiarity input is chosen randomly which has three membership functions as 'unfamiliar', 'medium' and 'familiar'. The second input has also three membership functions which are 'slow', 'medium' and 'high'. The output of Driver Fuzzy Controller has three membership functions which are 'weak','medium' and 'strong'. Concerning the Environment Fuzzy Controller, it has in turn two inputs which are departure time and weather information that are both of them chosen randomly because SUMO can't furnish their values. The first input represents the time of traveling within the day which has five membership functions as 'night', 'morning', 'midday', 'evening' and 'night'. The second input is the weather information which has three membership functions as 'bad', 'medium' and 'good'. Environment preference is the output of Environment Fuzzy Controller, its membership functions can be 'weak', 'medium' and 'strong'. Moreover, the others fuzzy controllers like PD (Path/Driver), DE (Driver/Environment) and PDE (Path/Driver/Environment) have all of them three membership functions which are 'weak', 'medium' and 'strong'. Further
TABLE IV

THE FuZzY RulES OF THE DRIVER FLC

\begin{tabular}{|c||c||c|c|}
\hline \multicolumn{1}{|c||}{ Rule no } & \multicolumn{2}{|c|}{ Inputs } & Output \\
\hline \multirow{2}{*}{1} & $\begin{array}{c}\text { Driver familiarity } \\
\text { with path i }\end{array}$ & $\begin{array}{c}\text { Usual-driving } \\
\text { speed }\end{array}$ & Driver preference \\
\hline 2 & Familiar & High & Strong \\
\hline 3 & Familiar & Medium & Strong \\
\hline 4 & Medium & Slow & Medium \\
\hline 5 & Medium & Medium & Strong \\
\hline 6 & Medium & Slow & Medium \\
\hline 7 & Unfamiliar & High & Weak \\
\hline 8 & Unfamiliar & Medium & Medium \\
\hline 9 & Unfamiliar & Slow & Medium \\
\hline
\end{tabular}

details about these fuzzy controllers are presented in previous research works [37], [60] and [61].

The method used for the fuzzy controllers' inference process is Mamdani(max-min). Table IV lists the fuzzy rules of the Driver Fuzzy Controller, based on the rules type if-then. In the majority of FLC applications, the forms of MFs are defined by humans arbitrarily. However, it cannot ensure providing the optimal control for the corresponding system. To promote the performance of the FLC, PSO technique is adopted to optimize the shapes of the MFs. At each step time, the PSO algorithm trains the FLC parameters of the final block (PDE Fuzzy Controller) since we do not have a suitable interpretable 


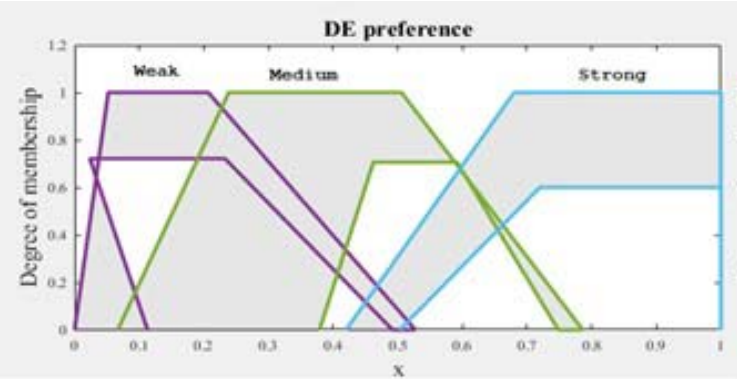

(a)

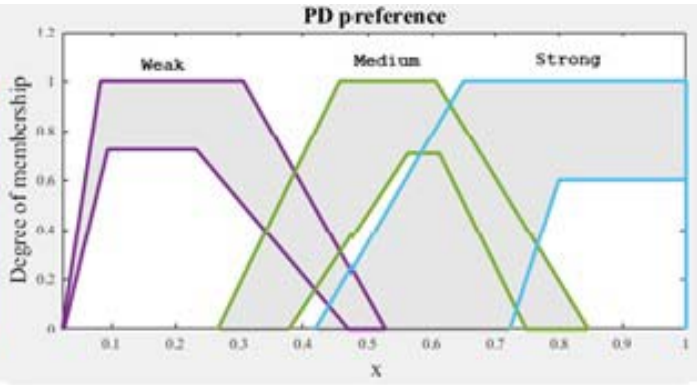

(b)

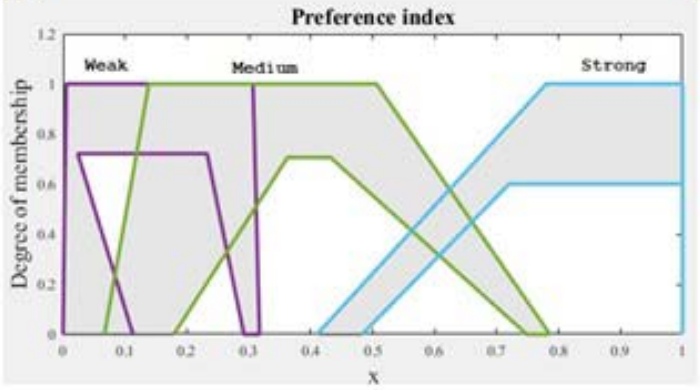

(c)

Fig. 4. Optimized Inputs (a)(b) and output (c) MFs of (PDE) FLC.

fitness function for the other Interval Type-2 Fuzzy blocks. The proposed FLC has two inputs and one output, each of them contains three MFs which have trapezoidal forms. Figure 4 shows the proposed FLC membership functions optimised by PSO.

To find the optimal MFs' adjustments, their correspondent search range, minimum and maximum values were initialized.

Table V illustrates the Interval Type-2 parameters of (PDE) FLC before PSO tuning process. Therefore, the purpose of PSO algorithm application in fine-tuning the membership functions of PDE Fuzzy Controller is to further help the driver to be well guided to his/her destination, while circumventing $\mathrm{jam} / \mathrm{congestion}$ situations. Hence, to find the candidate solution having the best performance, positions of all particles should be evaluated by fitness function.

The evaluation function used in our algorithm depended principally on a central criterion which is the density in the path. Our goal was to minimize the fitness function calculated as follows:

$$
\begin{aligned}
\text { Fitness } & =\frac{\text { Density of the lane }}{\sum \text { Density of possible lanes }} \\
\text { Density of the lane } & =\frac{\text { vehicle count }}{\text { length edge }}
\end{aligned}
$$

where Density of the lane designs the density of the planned next lane to reach among all the possible lanes to achieve the destination and calculated by the division between vehicle count and length edge during the simulation. Based on several experiments, we choose to fix the equation $5 c 1$ and $c 2$ 's parameters values equal to 2 , getting almost the best general performance. Besides, the size of population swarm is 100 and the maximal number of iterations is set to $\mathrm{NI}=50$ iterations. Nonetheless, regarding the inertia parameter $w$, it was chosen to be fixed to a value equal to 0,99 according to many tests as well.

\section{B. Road Traffic Simulations}

Traffic simulation is an important tool for modeling the operations of dynamic traffic systems. It helps analyzing the causes and potential solutions of traffic problems such as traffic jams, congestions, and traffic safety. We are interested in the microscopic type of simulation. There is a lot of traffic simulators available nowadays with different features, such as VISUM, Vissim, CORSIM, MATsim and SUMO [62].

In our research study, the simulator SUMO was chosen as it enables its users to load different road networks and set various traffic streams. SUMO helps to investigate several research topics like traffic light, route choice and communication simulation between vehicles [63]. It is thanks to its numerous benefits that SUMO is extensively used. Firstly, it is an open portable source for microscopic simulation of road traffic [20]. Furthermore, this package has the capacity to design both road network infrastructure and traffic demand.

A network file (.net.xml) comprises information concerning the map structure: nodes (junctions), edges (streets), and the connections between them. In fact, the network can be imported from OpenStreetMap (OSM). A route is a long trip, whose definition comprises the first and the last edges, along with all edges the car will pass. These routes are saved in a route file (.rou.xml). Supplementary files (.add.xml) can be added to SUMO information pertaining to the map or the traffic lights. The output of a SUMO simulation is recorded in a journey information file (.tripinfo.xml) that contains information relating to each car's departure time, the time the car waits to start at (offset), the time the car arrives, the travel time and the number of steps in which the car velocity is below $0.1 \mathrm{~m} / \mathrm{s}$ 
TABLE V

PARAmeters OF THE MEMBERship FunCtions

\begin{tabular}{|c|c|c|c|c|c|}
\hline \multicolumn{2}{|c|}{ Inputs/Outputs } & MF & Range & Min & Max \\
\hline \multirow{6}{*}{ I } & \multirow{3}{*}{ PD preference } & Weak & [a1,a2; b1,b2; c1,c2; d1,d2] & {$[0,0 ; 0,0 ; 0.25,0.15 ; 0.45,0.3]$} & {$[0,0 ; 0,0 ; 0.35,0.25 ; 0.55,0.4]$} \\
\hline & & Medium & {$[\mathrm{a} 1, \mathrm{a} 2 ; \mathrm{b} 1, \mathrm{~b} 2 ; \mathrm{c} 1, \mathrm{c} 2 ; \mathrm{d} 1, \mathrm{~d} 2]$} & {$[0.01,0.18 ; 0.32,0.48 ; 0.68,0.51 ; 0.88,0.72]$} & {$[0.11,0.28 ; 0.42,0.58 ; 0.78,0.61 ; 0.98,0.82]$} \\
\hline & & Strong & {$[\mathrm{a} 1, \mathrm{a} 2 ; \mathrm{b} 1, \mathrm{~b} 2 ; \mathrm{c} 1, \mathrm{c} 2 ; \mathrm{d} 1, \mathrm{~d} 2]$} & {$[0.4,0.6 ; 0.75,0.84 ; 1,1 ; 1,1]$} & {$[0.5,0.7 ; 0.85,0.94 ; 1,1 ; 1,1]$} \\
\hline & \multirow{3}{*}{ DE preference } & Weak & {$[\mathrm{a} 1, \mathrm{a} 2 ; \mathrm{b} 1, \mathrm{~b} 2 ; \mathrm{c} 1, \mathrm{c} 2 ; \mathrm{d} 1, \mathrm{~d} 2]$} & {$[0,0 ; 0,0 ; 0.3,0.15 ; 0.6,0.4]$} & {$[0,0 ; 0,0 ; 0.4,0.25 ; 0.7,0.5]$} \\
\hline & & Medium & {$[\mathrm{a} 1, \mathrm{a} 2 ; \mathrm{b} 1, \mathrm{~b} 2 ; \mathrm{c} 1, \mathrm{c} 2 ; \mathrm{d} 1, \mathrm{~d} 2]$} & {$[0.15,0.3 ; 0.37,0.4 ; 0.57,0.54 ; 0.75,0.7]$} & {$[0.25,0.4 ; 0.47,0.5 ; 0.67,0.64 ; 0.85,0.8]$} \\
\hline & & Strong & {$[\mathrm{a} 1, \mathrm{a} 2 ; \mathrm{b} 1, \mathrm{~b} 2 ; \mathrm{c} 1, \mathrm{c} 2 ; \mathrm{d} 1, \mathrm{~d} 2]$} & {$[0.4,0.6 ; 0.75,0.84 ; 1,1 ; 1,1]$} & {$[0.5,0.7 ; 0.85,0.94 ; 1,1 ; 1,1]$} \\
\hline \multirow{3}{*}{$\mathbf{O}$} & \multirow{3}{*}{ Preference index } & Weak & {$[\mathrm{a} 1, \mathrm{a} 2 ; \mathrm{b} 1, \mathrm{~b} 2 ; \mathrm{c} 1, \mathrm{c} 2 ; \mathrm{d} 1, \mathrm{~d} 2]$} & {$[0,0 ; 0,0 ; 0.3,0.15 ; 0.6,0.4]$} & {$[0,0 ; 0,0 ; 0.4,0.25 ; 0.7,0.5]$} \\
\hline & & Medium & {$[\mathrm{a} 1, \mathrm{a} 2 ; \mathrm{b} 1, \mathrm{~b} 2 ; \mathrm{c} 1, \mathrm{c} 2 ; \mathrm{d} 1, \mathrm{~d} 2]$} & {$[0.15,0.35 ; 0.35,0.5 ; 0.6,0.5 ; 0.873,0.75]$} & {$[0.25,0.45 ; 0.45,0.6 ; 0.7,0.6 ; 0.973,0.85]$} \\
\hline & & Strong & {$[\mathrm{a} 1, \mathrm{a} 2 ; \mathrm{b} 1, \mathrm{~b} 2 ; \mathrm{c} 1, \mathrm{c} 2 ; \mathrm{d} 1, \mathrm{~d} 2]$} & {$[0.4,0.6 ; 0.75,0.84 ; 1,1 ; 1,1]$} & {$[0.5,0.7 ; 0.85,0.94 ; 1,1 ; 1,1]$} \\
\hline
\end{tabular}

(temporal stops in driving). SUMO offers a high flexibility level, such that the user can find and modify the objects during simulation and permit the adaptation of the simulation online owing to TraCI (Traffic Control Interface) [64]. TraCIPython, TraCI4j (TraCI for Java) and TraCI4Matlab are three essential implementations of the TraCI protocol which allow the interaction with SUMO [65].

\section{Vi. Performance Evaluation of PSO BASED AHIT2FKRS}

The road traffic management necessitates a clear understanding of the flows, especially jam or congestion situations and it is very expensive to carry out the real plan.

That is why, the traffic simulation is the best recourse to test, to make predictions in a scientifically proven way and to evaluate different cases. Indeed, they demonstrate their ability to predict efficient solutions to complex problems like routing problem [17], [45], [66]. The routing problem consists in finding strategies of choosing itineraries to vehicles to minimize their travel time and reduce traffic jam on the road network. Thus, the route choice process is significant to enhance the traffic fluency situation and road quality. It pertains to the selection of alternative routes between origin and destination (O-D) in road networks. The simplest strategy found in literature is Dijkstra algorithm [67] that allows every vehicle to find the shortest route between every (O-D) pair. Although most contemporary routing algorithms allow tremendously fast computation of the shortest paths, they necessitate some preprocessing of the traffic network and storage of data which is produced from this preprocessing.

Therefore, this paper introduces a route guidance algorithm using both the shortest road information and that of jam/congestion on diverse paths. Before each intersection, the suggested PSO based AHIT2FKRS is carried out to choose the best following road to attain destination evading crowded areas, and thus conceding route length and time. The results of the proposed approach were compared with those of Dijkstra's algorithm (1959), HIT2FKRS, HIT1FKRS and those of HIT1FKRS optimized by PSO based on the average travel time and path flow criteria.

Table VI lists the routing algorithm. In this study, our proposal was tested with four wide and heterogeneous metropolitan areas with different total roads number, total intersections, traffic signals and varying number of vehicles situated in the cities of Sfax (Tayeb Mhiri zone), Luxembourg (LuST), Bologna and Cologne.
TABLE VI

ROUTING ALGORITHM

\begin{tabular}{l}
\hline Routing algorithm \\
\hline 1. Initialize the trip (Origin and Destination). \\
2. If vehicle $\mathrm{x}$ detect an intersection y \\
3. Look for the following possible links (roads) \\
4. Assess every subsequent possible road making use of the \\
evolutionary HIT2FLS \\
5. Compare the preference index of every road and choose \\
the best route which has the highest value of preference index
\end{tabular}

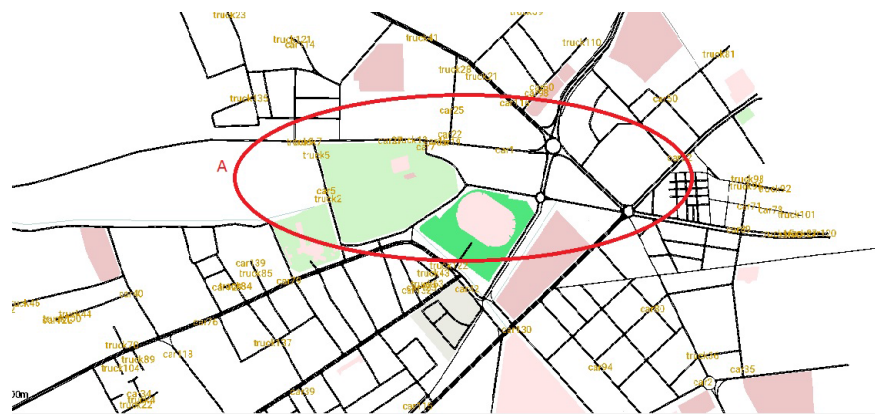

Fig. 5. Road network topology of Tayeb mhiri zone.

The characteristics of the chosen realistic traffic areas are presented as follows:

$\underline{\text { Scenario 1: }}$

The map of Tayeb Mhiri zone (Sfax, Tunisia) was imported from OSM and essentially edited using JOSM (Java OpenStreetMap Editor) before applying SUMO's Netconvert (Figure 5). SUMO includes three likely routing applications, namely DUAROUTER, JTRROUTER and DFROUTER, which allow to compute routes. In this scenario, we used the DUAROUTER application for generating traffic flow and routes. The area covered by this scenario is about $18 \mathrm{~km}^{2}$ with a total of 852 of roads. Table VII reveals information about the selected road network topology.

Scenario 2:

To have a realistic scenario, the area should be big enough to display the congestion patterns observable in contemporary cities. Thus, we chose the City of Luxembourg that covers an area of about $156 \mathrm{~km}^{2}$ with a total of $931 \mathrm{~km}$ of roads (Table VII). Figure 6 shows the topology of the LuST Scenario. The aim of using city's public transport database was to find information concerning bus itineraries. In this scenario, a total of 561 bus stops were introduced, with 38 bus itineraries inside the city for a total of 2240 buses per day [68]. 
TABLE VII

TOPOLOGY INFORMATION

\begin{tabular}{|c||c||c||c|c|}
\hline & Scenario1 & Scenario2 & Scenario3 & Scenario4 \\
\hline Area $\left(\mathrm{km}^{2}\right)$ & 18 & 156 & 25 & 400 \\
\hline $\begin{array}{c}\text { Total } \\
\text { junctions }\end{array}$ & 362 & 2365 & 1539 & 31614 \\
\hline Total roads & 852 & 5959 & 2856 & 71085 \\
\hline Traffic lights & 15 & 203 & 99 & 1219 \\
\hline $\begin{array}{c}\text { Vehicles } \\
\text { number }\end{array}$ & 500 & 2240 & 22000 & 700000 \\
\hline
\end{tabular}

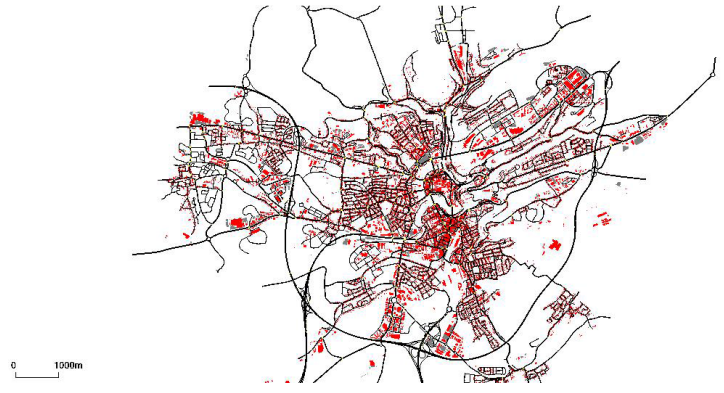

Fig. 6. LuST scenario topology.

Scenario 3:

Bologna Ringway Dataset models a realistic traffic scenario from the city of Bologna [69], Italy, during a typical day between $8 \mathrm{AM}$ and 9AM with more than 22000 vehicles in a $25 \mathrm{~km}^{2}$ area. Figure 7 reveals the topology of the Bologna Scenario. The traffic demand is defined only over one hour in a typical morning. This scenario covers only the main streets of the road network (Table VII).

The data was produced beginning from Induction Loops, amassed by the iTETRIS European collaborative project [70].

Scenario 4:

The "TAPAS Cologne" simulation scenario represents the traffic in Cologne city (Germany) for a period of 24 hours in a typical working day [71]. The Cologne city has around 400 square kilometers, comprising about 700000 individual car trips (Table VII). The information about the traffic demand on the macroscopic traffic flows across the Cologne urban area (the O-D matrix) is obtained via the methodology of Travel and Activity PAtterns Simulation (TAPAS). The traffic assignment of the vehicular flows described by the TAPASCologne O-D matrix over the road topology is achieved using Gawron's dynamic user assignment algorithm. Figure 8 shows the topology of the TAPASCologne Scenario.

\section{A. Results and Discussion}

The scalability and efficiency issues of our proposal were discussed in the scenario 4 (Cologne) using the urban traffic simulator (SUMO). In this case study, more than 10 simulation series were carried out with the change of the origins and destinations, departure time and congestion/jam positions. In addition, SUMO platform showed that it is possible to simulate in real time scenarios with more than 100.000 up to 200.000 vehicles with different right-of-way rules, traffic lights and lane changing [72]. Regarding scenario1, presented in Figure 5, a total of 500 cars were encompassed in the

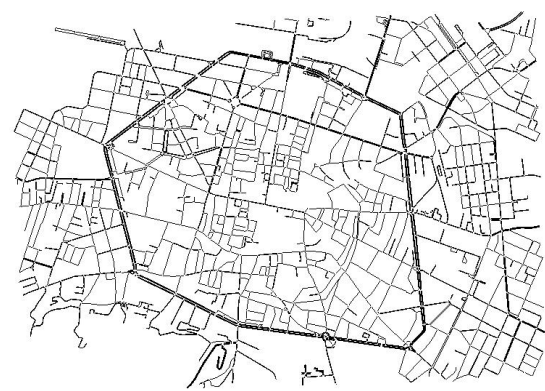

Fig. 7. Bologna scenario topology.

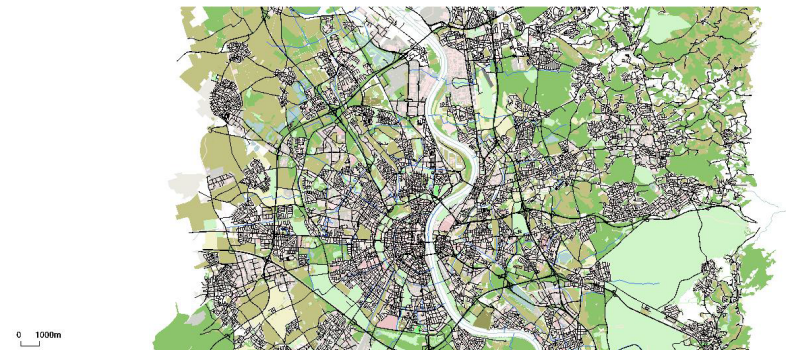

Fig. 8. TAPASCologne scenario topology.

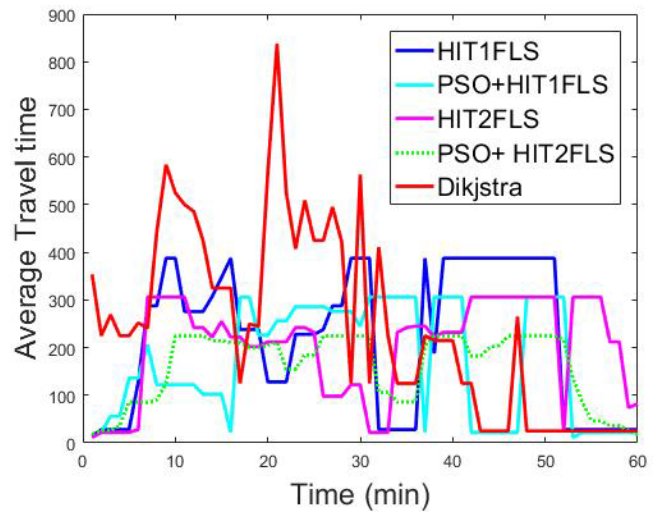

Fig. 9. Vehicles average travel time between O-D pairs (scenario 1).

simulation from various O-D pairs belonging to the same road network. In some roads of the network, the permitted speed is $20 \mathrm{~m} / \mathrm{s}$, and in others, it is $40 \mathrm{~m} / \mathrm{s}$, which is considered as reasonably elevated. Indeed, the enforcement of a congestion situation was realized by the simultaneous addition of an elevated number of vehicles in the closest roads. For this, 100 vehicles (travel demands) were added in area A of the considered scenario from 1000th to 1500th seconds.

As shown in Figures 9 and 10, the average travel time and the number of vehicles are more adjustable in the whole network. It is revealed in Figure 9 that due to congestion, Dijkstra's algorithm did not provide the shortest travel time. Using the proposed approach (PSO+AHIT2FLS), the travel time was assessed taking into account both distance and congestion. According to Table VIII, we suggested to the same car an itinerary which is found by two methods with the same conditions of road traffic simulation, considering the congested roads. It is noteworthy that the three possible paths 


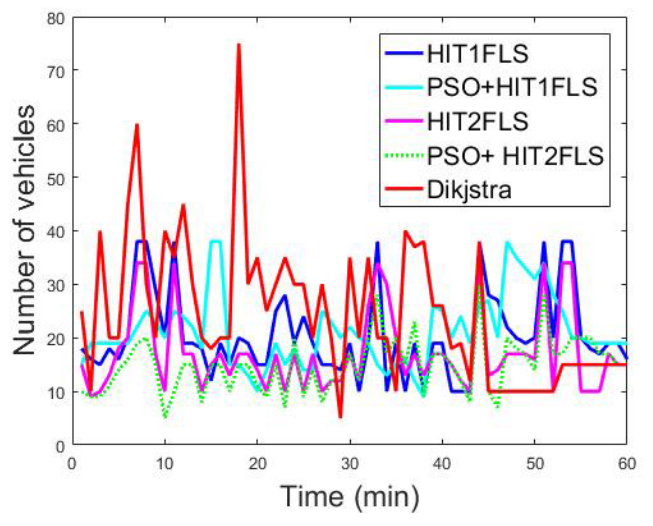

Fig. 10. Vehicles number circulating in the road network (scenario1).

TABLE VIII

Route SElECTION OF ONE CAR (From ORIGiN ROAD 30_4 TO DESTINATION ROAD 31_5 )

\begin{tabular}{|c||c||c||c|}
\hline Intersection & $\begin{array}{c}\text { Route selected } \\
\text { using Dijkstra's } \\
\text { algorithm }\end{array}$ & $\begin{array}{c}\text { Route selected } \\
\text { using } \\
\text { EHIT2FLS }\end{array}$ & $\begin{array}{c}\text { Other } \\
\text { possible } \\
\text { Route }\end{array}$ \\
\hline 1 & $30 \_3$ & $32 \_3$ & $30 \_3$ \\
\hline 2 & $30 \_2$ & $32 \_2$ & $30 \_2$ \\
\hline 3 & $31 \_0$ & $32 \_1$ & $30 \_1$ \\
\hline 4 & $31 \_1$ & $31 \_0$ & $30 \_0$ \\
\hline 5 & $31 \_2$ & $31 \_1$ & $29 \_7$ \\
\hline 6 & $31 \_3$ & $31 \_2$ & $29 \_6$ \\
\hline 7 & $31 \_4$ & $31 \_3$ & $29 \_5$ \\
\hline 8 & $31 \_5$ & $31 \_4$ & $29 \_4$ \\
\hline 9 & - & $31 \_5$ & $31 \_5$ \\
\hline Average speed(m/s) & 20 & 30 & 30 \\
\hline Travel time $\mathbf{( s )}$ & 970 & 738 & 1056 \\
\hline Distance $\mathbf{( m )}$ & 7050 & 9160 & 11040 \\
\hline
\end{tabular}

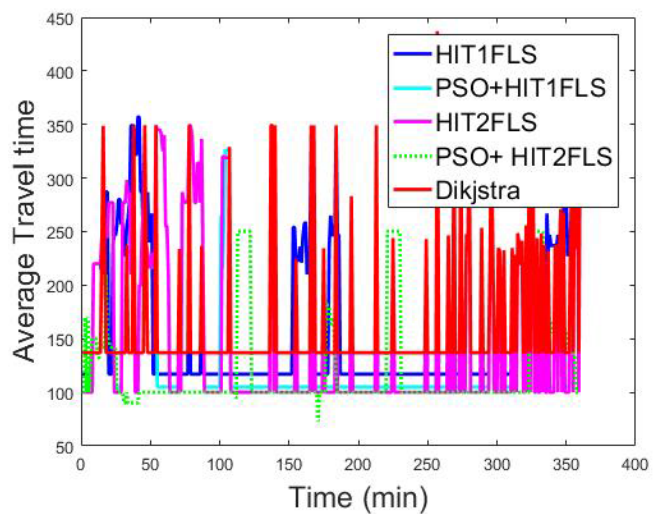

Fig. 11. Vehicles average travel time between O-D pairs (scenario2).

have not the same distance and the same average speed. The corresponding travel times of each path are diverse depending on the itinerary selection method. The obtained results confirmed the efficiency of the proposed method. To show that our system behaves well with many realistic traffic scenarios, we decided to apply it with European cities like Luxembourg, Bologna and Cologne. The scenarios must be sufficiently big to demonstrate the standard congestion patterns noticeable in contemporary cities. Figure 6 reveals the LuST Scenario topology, with streets colored by type with red for the main

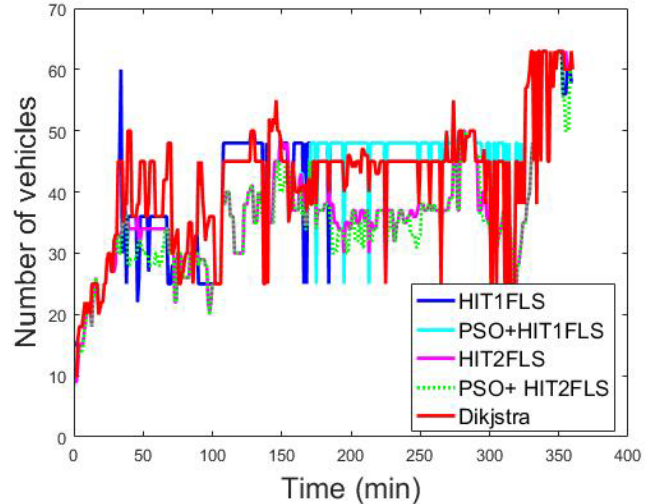

Fig. 12. Vehicles number circulating in the road network (scenario 2).

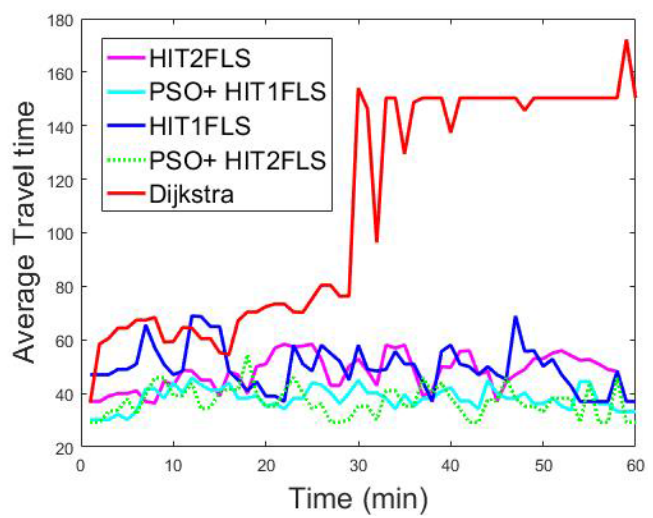

Fig. 13. Vehicles average travel time between O-D pairs (scenario3).

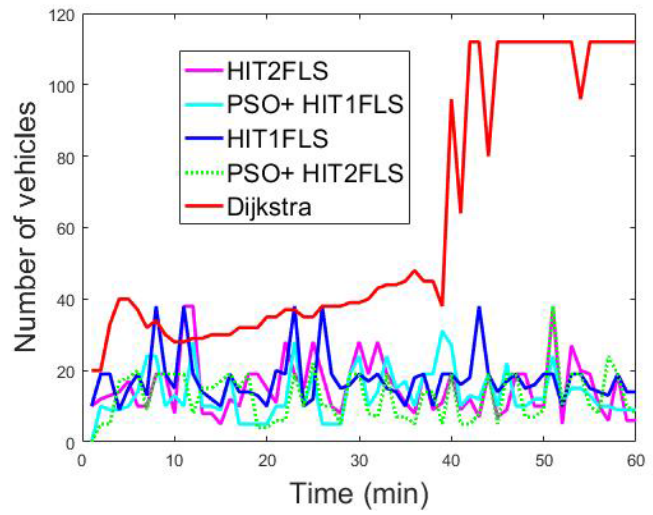

Fig. 14. Vehicles number circulating in the road network (scenario3).

arterial roads and black for residential roads. With respect to traffic flow quality in the road network, Figure 11 presents the advantages of the proposed PSO+AHIT2FLS, in the decrease of the average travel time in the whole road network during $6 h$, compared to the Dijkstra's method, to the HIT1FKRS, to the HIT2FLS, and to the HIT1FKRS optimized by PSO. For the third and fourth scenarios, concerning the cars number circulating in the road network, Figures 14 and 15, respectively, show that PSO+AHIT2FLS helps a high cars number reached their destinations early, compared to other methods. Besides, Figures 13 and 16 display the effectiveness of our system in 
TABLE IX

Mean of Average Travel Time (ATT) For Each Scenario

\begin{tabular}{|c|c|c|c|c|c|c|c|c|}
\hline \multirow{2}{*}{ Methods } & \multicolumn{2}{|c|}{ Scenario $1(3600 \mathrm{sc})$} & \multicolumn{2}{|c|}{ Scenario $2(21600 \mathrm{sc})$} & \multicolumn{2}{|c|}{ Scenario $3(3600 \mathrm{sc})$} & \multicolumn{2}{|c|}{ Scenario 4 (7201 sc) } \\
\hline & $\begin{array}{c}\text { Mean of } \\
\text { ATT }\end{array}$ & SD of ATT & $\begin{array}{c}\text { Mean of } \\
\text { ATT }\end{array}$ & SD of ATT & $\begin{array}{c}\text { Mean of } \\
\text { ATT }\end{array}$ & SD of ATT & $\begin{array}{c}\text { Mean of } \\
\text { ATT }\end{array}$ & SD of ATT \\
\hline M1: Dijkstra & 465.50 & 14.16 & $07.25 e+04$ & 900.94 & 112.51 & 05.83 & $01.47 \mathrm{e}+06$ & 764.49 \\
\hline M2: HIT1FLS & 193.49 & 13.07 & $06.63 e+04$ & 599.29 & 44.30 & 0.51 & $01.22 \mathrm{e}+06$ & 424.26 \\
\hline M3: PSO+HIT1FLS & 175.78 & 9.84 & $06.32 \mathrm{e}+04$ & 287.84 & 35.29 & 0.28 & $01.14 \mathrm{e}+06$ & 299.81 \\
\hline M4: HIT2FLS & 146.67 & 4.02 & $06.27 \mathrm{e}+04$ & 328.26 & 44.18 & 0.34 & $01.21 \mathrm{e}+06$ & 331.49 \\
\hline M5: PSO+AHIT2FLS & 96.40 & 3.68 & $06.25 \mathrm{e}+04$ & 174.80 & 34.95 & 0.18 & $01.13 e+06$ & 293.44 \\
\hline
\end{tabular}

TABLE $X$

ANova Results for Mean of Average Travel Time

\begin{tabular}{|l|l|l|l|l|l|l|l|l|l|l|l|l|}
\hline \multirow{2}{*}{ Methods } & \multicolumn{3}{|c|}{ Scenario 1 (3600 sc) } & \multicolumn{3}{c|}{ Scenario 2 (21600 sc) } & \multicolumn{3}{c|}{ Scenario 3 (3600 sc) } & \multicolumn{3}{c|}{ Scenario 4 (7201 sc) } \\
\cline { 2 - 13 } & lwr & upr & p-value & lwr & upr & p-value & lwr & upr & p-value & lwr & upr & p-value \\
\hline M1 vs M2 & 272.01 & 286.11 & $9.92 \mathrm{e}-09$ & 6273 & $6.94 \mathrm{e}+03$ & $9.92 \mathrm{e}-09$ & 68.21 & 71.55 & $9.92 \mathrm{e}-09$ & 247000 & $2.47 \mathrm{e}+05$ & $9.92 \mathrm{e}-09$ \\
\hline M1 vs M3 & 289.71 & 303.81 & $9.92 \mathrm{e}-09$ & $9.29 \mathrm{e}+03$ & $9.96 \mathrm{e}+03$ & $9.92 \mathrm{e}-09$ & 77.22 & 80.56 & $9.92 \mathrm{e}-09$ & 328710 & $3.29 \mathrm{e}+05$ & $9.92 \mathrm{e}-09$ \\
\hline M1 vs M4 & 318.82 & 332.92 & $9.92 \mathrm{e}-09$ & $9.85 \mathrm{e}+03$ & $1.05 \mathrm{e}+04$ & $9.92 \mathrm{e}-09$ & 68.32 & 71.67 & $9.92 \mathrm{e}-09$ & 260690 & $2.61 \mathrm{e}+05$ & $9.92 \mathrm{e}-09$ \\
\hline M1 vs M5 & 369.09 & 383.19 & $9.92 \mathrm{e}-09$ & $1.00 \mathrm{e}+04$ & $1.07 \mathrm{e}+04$ & $9.92 \mathrm{e}-09$ & 77.56 & 80.90 & $9.92 \mathrm{e}-09$ & 335900 & $3.36 \mathrm{e}+05$ & $9.92 \mathrm{e}-09$ \\
\hline M2 vs M3 & 17.70 & 31.80 & 0.0074 & $3.02 \mathrm{e}+03$ & $3.69 \mathrm{e}+03$ & $9.92 \mathrm{e}-09$ & 9.01 & 12.34 & $2.04 \mathrm{e}-08$ & 81710 & $8.23 \mathrm{e}+04$ & $9.92 \mathrm{e}-09$ \\
\hline M2 vs M4 & 46.81 & 60.91 & $9.95 \mathrm{e}-09$ & $3.58 \mathrm{e}+03$ & $4.25 \mathrm{e}+03$ & $9.92 \mathrm{e}-09$ & 0.113 & 3.45 & $\mathbf{1 . 0 0}$ & 13690 & $1.42 \mathrm{e}+04$ & $9.92 \mathrm{e}-09$ \\
\hline M2 vs M5 & 97.08 & 111.19 & $9.92 \mathrm{e}-09$ & $3.78 \mathrm{e}+03$ & $4.45 \mathrm{e}+03$ & $9.92 \mathrm{e}-09$ & 9.34 & 12.68 & $1.39 \mathrm{e}-08$ & 88900 & $8.94 \mathrm{e}+04$ & $9.92 \mathrm{e}-09$ \\
\hline M3 vs M4 & 29.11 & 43.21 & $4.79 \mathrm{e}-06$ & 561.80 & $1.23 \mathrm{e}+03$ & $\mathbf{0 . 1 4}$ & -8.89 & -5.55 & $2.45 \mathrm{e}-08$ & -68020 & $-6.74 \mathrm{e}+04$ & $9.92 \mathrm{e}-09$ \\
\hline M3 vs M5 & 79.38 & 93.48 & $9.92 \mathrm{e}-09$ & 758.80 & $1.43 \mathrm{e}+03$ & 0.019 & 0.33 & 3.68 & $\mathbf{0 . 9 9}$ & 7190 & $7.77 \mathrm{e}+03$ & $9.92 \mathrm{e}-09$ \\
\hline M4 vs M5 & 50.27 & 64.37 & $9.92 \mathrm{e}-09$ & 197 & 867.49 & $\mathbf{0 . 9 1}$ & 9.23 & 12.57 & $1.54 \mathrm{e}-08$ & 75210 & $7.58 \mathrm{e}+04$ & $9.92 \mathrm{e}-09$ \\
\hline
\end{tabular}

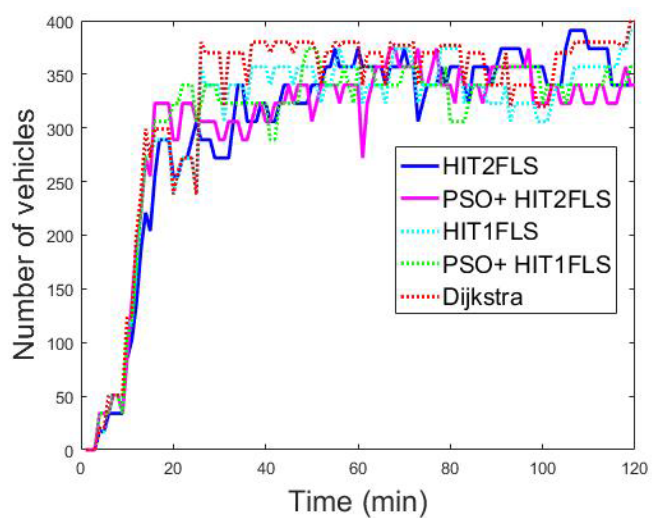

Fig. 15. Vehicles number circulating in the road network (scenario4).

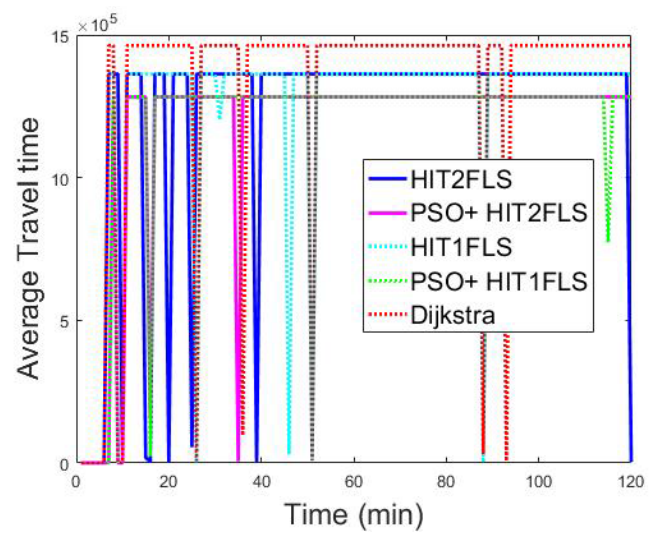

Fig. 16. Vehicles average travel time between O-D pairs (scenario4).

terms of average travel time, compared to the other methods. For a better comparison, we resume results in a quantitative and statistical way presented in Table IX, by measuring both the mean of average travel time and standard deviation. The standard deviation is calculated as follows:

$$
S D=\sqrt{\frac{\sum_{i=1}^{i=n}\left(X_{i}-\overline{X_{i}}\right)^{2}}{n}}
$$

where $\mathrm{n}$ is the number of simulations which is equal to 10 in our case, $X_{i}$ indicates the Average Travel Time (ATT) for simulation $i$ and $\overline{X_{i}}$ indicates the mean of ATT.

In fact, Table IX affirms the global results illustrated by Figures (9-16). For all scenarios, the values of the ATT's means and ATT's SD of our proposal (PSO-AHIT2FLS) are lower than those computed using the other methods.

Since the results shown in Table IX are not sufficient to decide about the best method, a statistical analysis is required to have better discussions of the results and study the differences between the means computed from the average travel time (ATT) of 10 different simulations for each method and each scenario. We applied the standard analysis of variance (ANOVA) test and performed the mean equality test by using Tukey's HSD (Honestly Significant Difference) test [73]. Table X presents the results of the ANOVA test. In this table, two means are considered as different if the p-value is less than $5 \%$.

As a conclusion from this analysis, and based on the means values, method M5 (PSO+HIT2FLS) is better than method M4 (HIT2FLS) for the scenarios 1, 3, and 4. However, statistically, the difference between the two methods is not significant in scenario 2 ( $\mathrm{p}$-value $=0.91>0.05$ ). Moreover, method M5 is better than the method M3 based on the means values for the scenarios 1,2 , and 4 . But, statistically, there is no significant differences in the scenario 3 (pvalue $=0.99>0.05$ ). Tables IX and $X$ reveal the improvements using PSO for the learning of HIT2FKRS in terms of average 
travel time compared to the following methods: Dijkstra, HIT2FKRS, HIT1FKRS, and HIT1FKRS optimized by PSO. Therefore, about $45 \%$ of cars have altered their routes after the implementation of PSO+AHIT2FLS. The simulation results comprise the impact of the six above-mentioned external factors: density, maximum allowed speed in the path, driver familiarity with the path, usual driving speed, departure time and weather information. These findings affirm the significant impact of the chosen contextual factors and the efficacy of the proposed PSO-based AHIT2FLS in improving the traffic network quality and circumventing the states of congestion or jam.

\section{CONCLUSION}

In this paper, a PSO based Adaptive Hierarchical Interval Type-2 Fuzzy Knowledge Representation System for Travel Route Guidance was presented. This advanced traffic management system allows an intelligent and prompt adjustment of the road traffic in the network depending on the real-time changes. The route is selected according to the quality of traffic and route length, together with contextual factors pertaining to the driver, the environment and the path incorporated in a hierarchical fuzzy model. Actually, the proposed system contributes to an improvement in traffic fluency (the road network is enabled to support a much elevated number of cars without reducing the vehicles average speed) while taking into consideration the real-time road traffic information. Thanks to this system, the traffic congestion situations number has been reduced by evading the extensive use of the same road at the same time (suggesting routes with lower travel time). For this study, four big realistic traffic scenarios situated in the cities of Sfax, Luxembourg, Bologna and Cologne have been tested while using SUMO, a recognized traffic simulator. Simulation results affirm that the suggested system (PSO based AHIT2FKRS) provides a better road traffic quality on the whole road network without a great loss on individual travel time compared to the Dijkstra method, HIT2FKRS, HIT1FKRS and HIT1FKRS optimized by PSO. Furthermore, our PSO based AHIT2FKRS can ameliorate the cars number that attain their destination and the mean travel time. As a future avenue, we plan to provide communications among nearby vehicles in order to know the state of network in a global way (accidents/Jams). Taking advantage of $\mathrm{V} 2 \mathrm{~V}$ (Vehicle to Vehicle) communications for traffic management with the effectiveness of our PSO based AHIT2FLS, we can offer good road traffic quality of all regions of city.

\section{REFERENCES}

[1] L. Wismans, E. V. Berkum, and M. Bliemer, "Effects of optimizing externalities using cooperating dynamic traffic management measures on network level," J. Intell. Transp. Syst., vol. 17, no. 1, pp. 65-77, Jan. 2013.

[2] H. M. Kammoun, I. Kallel, J. Casillas, A. Abraham, and A. M. Alimi, "Adapt-Traf: An adaptive multiagent road traffic management system based on hybrid ant-hierarchical fuzzy model," Transp. Res. C, Emerg. Technol., vol. 42, pp. 147-167, May 2014.

[3] A. M. de Souza, C. A. Brennand, R. S. Yokoyama, E. A. Donato, E. R. Madeira, and L. A. Villas, "Traffic management systems: A classification, review, challenges, and future perspectives," Int. J. Distrib. Sensor Netw., vol. 13, no. 4, Apr. 2017, Art. no. 155014771668361.
[4] M. Hajiahmadi, V. L. Knoop, B. De Schutter, and H. Hellendoorn, "Optimal dynamic route guidance: A model predictive approach using the macroscopic fundamental diagram," in Proc. 16th Int. IEEE Conf. Intell. Transp. Syst. (ITSC), Oct. 2013, pp. 1022-1028.

[5] J. Pan, I. S. Popa, K. Zeitouni, and C. Borcea, "Proactive vehicular traffic rerouting for lower travel time," IEEE Trans. Veh. Technol., vol. 62 , no. 8, pp. 3551-3568, Oct. 2013.

[6] C. Li, S. G. Anavatti, and T. Ray, "Analytical hierarchy process using fuzzy inference technique for real-time route guidance system," IEEE Trans. Intell. Transp. Syst., vol. 15, no. 1, pp. 84-93, Feb. 2014.

[7] J. Lin, W. Yu, X. Yang, Q. Yang, X. Fu, and W. Zhao, "A realtime en-route route guidance decision scheme for transportation-based cyberphysical systems," IEEE Trans. Veh. Technol., vol. 66, no. 3, pp. 2551-2566, Mar. 2017.

[8] P. G. Balaji and D. Srinivasan, "Type-2 fuzzy logic based urban traffic management," Eng. Appl. Artif. Intell., vol. 24, no. 1, pp. 12-22, Feb. 2011.

[9] H. Hagras and C. Wagner, "Towards the wide spread use of type-2 fuzzy logic systems in real world applications," IEEE Comput. Intell. Mag., vol. 7, no. 3, pp. 14-24, Aug. 2012.

[10] S. Cherif, N. Baklouti, V. Snasel, and A. M. Alimi, "New fuzzy similarity measures: From intuitionistic to type-2 fuzzy sets," in Proc. IEEE Int. Conf. Fuzzy Syst. (FUZZ-IEEE), Jul. 2017, pp. 1-6.

[11] N. Baklouti and A. M. Alimi, "Type-2 fuzzy logic controller design using a real-time (PSO) algorithm applied to "iRobot create robot," Int. J. Robot. Autom., vol. 32, no. 2, pp. 117-126, 2017.

[12] Y. Ghozzi, N. Baklouti, and A. M. Alimi, "A beta-fuzzy-near-sets approach to research for visually similar content images," in Proc. IEEE Int. Conf. Fuzzy Syst. (FUZZ-IEEE), Jul. 2017, pp. 1-6.

[13] F. Herrera, M. Lozano, and J. L. Verdegay, "A learning process for fuzzy control rules using genetic algorithms," Fuzzy Sets Syst., vol. 100, nos. 1-3, pp. 143-158, Nov. 1998.

[14] C. H. Tan, K. S. Yap, and H. J. Yap, "Application of genetic algorithm for fuzzy rules optimization on semi expert judgment automation using pittsburg approach," Appl. Soft Comput., vol. 12, no. 8, pp. 2168-2177, Aug. 2012.

[15] Y.-H. Chang, C.-W. Chang, C.-W. Tao, H.-W. Lin, and J.-S. Taur, "Fuzzy sliding-mode control for ball and beam system with fuzzy ant colony optimization," Expert Syst. Appl., vol. 39, no. 3, pp. 3624-3633, Feb. 2012.

[16] R. Eberhart and J. Kennedy, "A new optimizer using particle swarm theory," in Proc. Symp. Micro Mach. Hum. Sci., 1995, pp. 39-43.

[17] Y.-J. Gong and J. Zhang, "Real-time traffic signal control for roundabouts by using a PSO-based fuzzy controller," in Proc. IEEE Congr. Evol. Comput. (CEC), Jun. 2012, pp. 1-8.

[18] W. Elloumi, H. El Abed, A. Abraham, and A. M. Alimi, "A comparative study of the improvement of performance using a PSO modified by ACO applied to TSP," Appl. Soft Comput., vol. 25, pp. 234-241, Dec. 2014.

[19] N. Chouikhi, B. Ammar, N. Rokbani, and A. M. Alimi, "PSO-based analysis of echo state network parameters for time series forecasting," Appl. Soft Comput., vol. 55, pp. 211-225, Jun. 2017.

[20] D. Krajzewicz, G. Hertkorn, C. Rossel, and P. Wagner, "SUMO (simulation of urban mobility) - An open-source traffic simulation," in Proc. 4th Middle East Symp. Simulation Modeling, 2002, pp. 183-187.

[21] E. Avineri, "Soft computing applications in traffic and transport systems: A review," in Soft Computing: Methodologies and Applications. Berlin, Germany: Springer, 2005, pp. 17-25.

[22] L. Fu, "An adaptive routing algorithm for in-vehicle route guidance systems with real-time information," Transp. Res. B, Methodol., vol. 35, no. 8, pp. 749-765, Sep. 2001.

[23] S. Peeta and J. W. Yu, "Adaptability of a hybrid route choice model to incorporating driver behavior dynamics under information provision," IEEE Trans. Syst., Man, Cybern. A, Syst. Humans, vol. 34, no. 2, pp. 243-256, Mar. 2004.

[24] Y. Wang, M. Papageorgiou, G. Sarros, and W. J. Knibbe, "Real-time route guidance for large-scale express ring-roads," in Proc. IEEE Intell. Transp. Syst. Conf., Sep. 2006, pp. 224-229.

[25] J. W. Ding, C. F. Wang, F. H. Meng, and T. Y. Wu, "Real-time vehicle route guidance using vehicle-to-vehicle communication," IET Commun., vol. 4, pp. 870-883, 2010.

[26] S. Bekhor, M. E. Ben-Akiva, and M. S. Ramming, "Adaptation of logit kernel to route choice situation," Transp. Res. Rec. J. Transp. Res. Board, vol. 1805, no. 1, pp. 78-85, Jan. 2002.

[27] S. L. Ben-Akiva, Discrete Choice Analysis: Theory and Applications to Travel Demand. Cambridge, MA, USA: MIT, 1985. 
[28] D. Teodorović, "Fuzzy logic systems for transportation engineering: The state of the art," Transp. Res. A, Policy Pract., vol. 33, no. 5, pp. 337-364, Jun. 1999.

[29] X. Zhang, E. Onieva, A. Perallos, E. Osaba, and V. C. S. Lee, "Hierarchical fuzzy rule-based system optimized with genetic algorithms for short term traffic congestion prediction," Transp. Res. C, Emerg. Technol., vol. 43, pp. 127-142, Jun. 2014.

[30] L. Nassar and F. Karray, "Fuzzy logic in VANET context aware congested road and automatic crash notification," in Proc. IEEE Int. Conf. Fuzzy Syst. (FUZZ-IEEE), Jul. 2016, pp. 1031-1037.

[31] L. Caggiani, R. Camporeale, and M. Ottomanelli, "A real time multiobjective cyclists route choice model for a bike-sharing mobile application," in Proc. 5th IEEE Int. Conf. Models Technol. Intell. Transp. Syst. (MT-ITS), Jun. 2017, pp. 645-650.

[32] J. M. Mendel, Uncertain Rule Based Fuzzy Logic Systems: Introduction and New Directions. Upper Saddle River, NJ, USA: Prentice-Hall, 2011.

[33] N. N. Karnik, J. M. Mendel, and Q. Liang, "Type-2 fuzzy logic systems," IEEE Trans. Fuzzy Syst., vol. 7, no. 6, pp. 643-658, 1999.

[34] B. Sabetghadam, F. Shabaninia, M. Vaziri, and S. Vadhava, "Type-2 fuzzy multiagent traffic signal control," in Proc. IEEE 13th Int. Conf. Inf. Reuse Integr. (IRI), Aug. 2012, pp. 509-512.

[35] Y. Bi, D. Srinivasan, X. Lu, Z. Sun, and W. Zeng, "Type-2 fuzzy multiintersection traffic signal control with differential evolution optimization," Expert Syst. Appl., vol. 41, no. 16, pp. 7338-7349, Nov. 2014.

[36] R. Li, C. Jiang, F. Zhu, and X. Chen, "Traffic flow data forecasting based on interval type-2 fuzzy sets theory," IEEE/CAA J. Automatica Sinica, vol. 3, no. 2, pp. 141-148, Apr. 2016.

[37] M. Zouari, N. Baklouti, H. Kammoun, J. Sanchez-Medina, M. B. Ayed, and A. M. Alimi, "Hierarchical interval type-2 beta fuzzy knowledge representation system for path preference planning," in Proc. IEEE Int. Conf. Fuzzy Syst. (FUZZ-IEEE), Jul. 2017, pp. 1-6.

[38] Y. Bi, X. Lu, Z. Sun, D. Srinivasan, and Z. Sun, "Optimal type-2 fuzzy system for arterial traffic signal control," IEEE Trans. Intell. Transp. Syst., vol. 19, no. 9, pp. 3009-3027, Sep. 2018.

[39] D. Teodorović, "Swarm intelligence systems for transportation engineering: Principles and applications," Transp. Res. C, Emerg. Technol., vol. 16, no. 6, pp. 651-667, Dec. 2008.

[40] A. A. Kazharov and V. M. Kureichik, "Ant colony optimization algorithms for solving transportation problems," J. Comput. Syst. Sci. Int., vol. 49, no. 1, pp. 30-43, Feb. 2010.

[41] E. Gurpreet and D. Vijay, "Open vehicle routing problem by ant colony optimization," Int. J. Adv. Comput. Sci. Appl., vol. 5, no. 3, pp. 63-68, 2014.

[42] J. Garcia-Nieto, A. C. Olivera, and E. Alba, "Optimal cycle program of traffic lights with particle swarm optimization," IEEE Trans. Evol. Comput., vol. 17, no. 6, pp. 823-839, Dec. 2013.

[43] C. C. You and L. H. Hyol, "Distributed traffic signal control using PSO based on probability model for traffic jam," in Proc. 12th Int. Conf. Intell. Autonomous Syst. (IAS), 2013, pp. 629-639.

[44] B. Wu and D. Wang, "Traffic signal networks control optimize with PSO algorithm," in Proc. 12th Int. Conf. Natural Comput., Fuzzy Syst. Knowl. Discovery (ICNC-FSKD), Aug. 2016, pp. 230-234.

[45] C. El Hatri and J. Boumhidi, "Traffic management model for vehicle re-routing and traffic light control based on multi-objective particle swarm optimization," Intell. Decis. Technol., vol. 11, no. 2, pp. 199-208, Jun. 2017

[46] Q. Li, W. Shangguan, B. Cai, and L. Chai, "Traffic flow guidance and optimization of connected vehicles based on swarm intelligence," in Proc. Chin. Control Conf. (CCC), Jul. 2019, pp. 2099-2104.

[47] J. Kennedy and R. C. Eberhart, Swarm Intelligence. San Mateo, CA, USA: Morgan Kaufmann, 2001.

[48] K. E. Parsopoulos and F. M. Vrahatis, "Unified particle swarm optimization for solving constrained engineering optimization problems," in Advances in Natural Computation. Springer, 2005, pp. 582-591.

[49] J. Garcia-Nieto and E. Alba, "Automatic parameter tuning with metaheuristics of the AODV routing protocol for vehicular ad-hoc networks," in Applications of Evolutionary Computation, D. C. Cecilia, B. Anthony, D. C. Gianni, E. Marc, F. Muddassar, F. Andreas, J. Grahl, G. Greenfield, M. Penousal, O. N. Michael, E. Tarantino, and N. Urquhart, Eds. Berlin, Germany: Springer, 2010, pp. 21-30.

[50] S. Bousnina, B. Ammar, N. Baklouti, and A. M. Alimi, "Learning system for mobile robot detection and tracking," in Proc. Int. Conf. Commun. Inf. Technol. (ICCIT), Jun. 2012, pp. 384-389.

[51] N. Baklouti and A. M. Alimi, "Real time PSO based adaptive learning type-2 fuzzy logic controller design for the iRobot create robot," in Proc. Int. Conf. Individual Collective Behaviors Robot. (ICBR), Dec. 2013, pp. $15-20$
[52] N. Baklouti, H. A. Lamti, K. Salhi, and A. M. Alimi, "PSO based adaptive learning fuzzy logic controller for the irobot create robot," in Proc. 13th Int. Conf. Hybrid Intell. Syst. (HIS), Dec. 2013, pp. 99-104.

[53] J. García-Nieto, J. Toutouh, and E. Alba, "Automatic tuning of communication protocols for vehicular ad hoc networks using metaheuristics," Eng. Appl. Artif. Intell., vol. 23, no. 5, pp. 795-805, Aug. 2010.

[54] L. A. Zadeh, "The concept of a linguistic variable and its application to approximate reasoning-1," Inf. Sci., vol. 8, no. 3, pp. 199-249, 1975.

[55] N. N. Karnik and J. M. Mendel, "Centroid of a type-2 fuzzy set," Inf. Sci., vol. 132, nos. 1-4, pp. 195-220, Feb. 2001.

[56] H. Hagras, "Embedding computational intelligence in pervasive spaces," IEEE Pervas. Comput., vol. 6, no. 3, pp. 85-89, Jul. 2007.

[57] O. Castillo and P. Melin, "A review on interval type-2 fuzzy logic applications in intelligent control," Inf. Sci., vol. 279, pp. 615-631, Sep. 2014

[58] M.-L. Lee, H.-Y. Chung, and F.-M. Yu, "Modeling of hierarchical fuzzy systems," Fuzzy Sets Syst., vol. 138, no. 2, pp. 343-361, Sep. 2003.

[59] W. Rattasiri and S. K. Halgamuge, "Computationally advantageous and stable hierarchical fuzzy systems for active suspension," IEEE Trans. Ind. Electron., vol. 50, no. 1, pp. 48-61, Feb. 2003.

[60] H. Lajmi, H. M. Kammoun, M. Zouari, A. M. Alimi, and J. M. Rodriguez, "Type-2-fuzzy rule base system based on ECUs communication in a smart city vehicular environment," in Proc. Int. Conf. Adv. Syst. Electr. Technol. (IC_ASET), Jan. 2017, pp. 460-466.

[61] M. Zouari, S. Cherif, H. Kammoun, H. Lajmi, and A. M. Alimi, "Towards type-2 fuzzy rule base system for road choice," in Proc. 15th Int. Conf. Intell. Syst. Design Appl. (ISDA), Dec. 2015, pp. 243-248.

[62] F. Jimínez, "Chapter 10-Simulation tools," in Intelligent Vehicles. London, U.K.: Butterworth, 2018, pp. 395-436.

[63] G. Soares, J. Macedo, Z. Kokkinogenis, and R. Rossetti, "An integrated framework for multi-agent traffic simulation using sumo and jade," in Proc. 1st SUMO User Conf. (SUMO), 2013, pp. 1-6.

[64] Introduction to TraCI. Accessed: 2008. [Online]. Available: http://sumo.dlr.de/wiki/TraCI

[65] A. Andres, F. Espinosa, E. Jorge, and E. Jairo, "TraCI4MATLAB: Enabling the integration of the SUMO road traffic simulator and MATLAB through a software re-engineering process," in Modeling Mobility With Open Data. 2015, pp. 155-170.

[66] J. Shi, C. Peng, Q. Zhu, P. Duan, Y. Bao, and M. Xie, "There is a will there is a way: A new mechanism for traffic control based on VTL and VANET,' in Proc. IEEE 16th Int. Symp. High Assurance Syst. Eng., Jan. 2015, pp. 240-246.

[67] E. W. Dijkstra, "A note on two problems in connexion with graphs," Numerische Math., vol. 1, no. 1, pp. 269-271, Dec. 1959.

[68] Luxembourg SUMO Traffic (LuST) Scenario. Accessed: 2015. [Online]. Available: https://github.com/lcodeca/LuSTScenario

[69] L. Bedogni, M. Gramaglia, A. Vesco, M. Fiore, J. Härri, and F. Ferrero, "The bologna ringway dataset: Improving road network conversion in SUMO and validating urban mobility via navigation services," IEEE Trans. Veh. Technol., vol. 64, no. 12, pp. 5464-5476, Dec. 2015.

[70] The Bologna Ringway Dataset. Accessed: 2014. [Online]. Available: https://sourceforge.net/projects/sumo/files/trafficdata/scenarios/ Bolognasmall

[71] TAPAS Cologne Scenario. Accessed: 2008. [Online]. Available: http://sumo.dlr.de/wiki/Data/Scenarios/TAPASCologne

[72] P. A. Lopez et al., "Microscopic traffic simulation using SUMO," in Proc. 21st Int. Conf. Intell. Transp. Syst. (ITSC), Nov. 2018, pp. 2575-2582.

[73] S. Morrison, J. Sosnoff, K. Heffernan, S. Jae, and B. Fernhall, "Aging, hypertension and physiological tremor: The contribution of the cardioballistic impulse to tremorgenesis in older adults," $J$. Neurological Sci., vol. 326, nos. 1-2, pp. 82-108, 2013

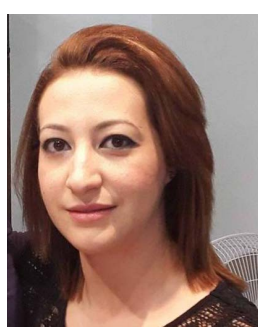

Mariam Zouari (Student Member, IEEE) received the M.S. degree from the Faculty of Economic Sciences and Management of Sfax (FSEGS) in 2012. She is currently pursuing the Ph.D. degree in computer systems engineering with the National Engineering School of Sfax (ENIS), University of Sfax. She is also a member of the Research Groups in Intelligent Machines (REGIM). Her research interests include knowledge representation, intelligent transportation system, neural fuzzy systems, and interval type-2 fuzzy logic systems. 


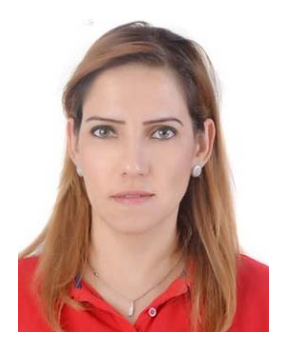

Nesrine Baklouti (Senior Member, IEEE) graduated in electrical engineering in 2004. She received the $\mathrm{Ph} . \mathrm{D}$. degree in electrical and computer engineering in 2013. She is currently a member of the REGIM-Lab on Intelligent Machines, University of Sfax. Her research interests include artificial intelligence, analysis and intelligent control, and robotic systems.

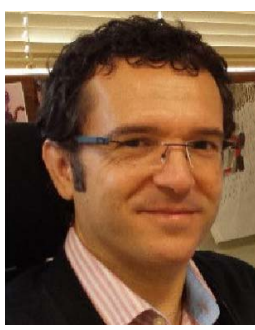

Javier Sanchez-Medina (Senior Member, IEEE) received the M.Eng. degree from the Telecommunications Faculty in 2002 and the Ph.D. degree from the Computer Science Department in 2008. He is interested in the application of evolutionary and parallel computing techniques to intelligent transportation systems. He has more than 20 international conference papers and 10 international journal articles. He is also very active as a Volunteer of the IEEE ITS Society, where he has been serving in a number of different positions. He is currently the Editor-in-Chief of the ITS Podcast and the ITS Newsletter, the Vice-President of the IEEE ITSS's Spanish Chapter, and the General Chair for the IEEE ITSC 2015.

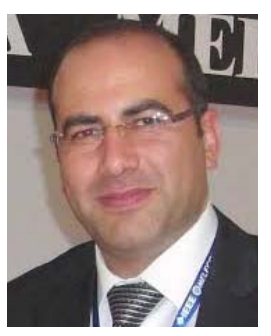

Habib M. Kammoun (Senior Member, IEEE) received the M.Sc. and Ph.D. degrees in computer science from the National Engineering School of Sfax (ENIS), University of Sfax, Tunisia, in 2005 and 2015, respectively. He is currently an Assistant Professor with the Department of Computer Science, Faculty of Sciences, University of Sfax. He is also a member of the Research Groups in Intelligent Machines (REGIM-Laboratory), University of Sfax, and the Machine Intelligence Research Labs (MIR Labs), USA. His research interests include soft computing, computational intelligence, intelligent transportation systems, and smart cities.

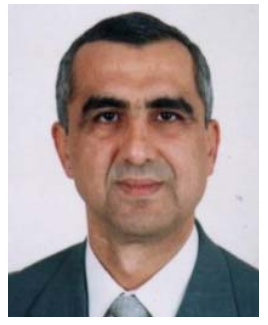

Mounir Ben Ayed (Senior Member, IEEE) received the Habilitation degree in computer system engineering from the Engineering School of Sfax, Tunisia, in 2013, the Ph.D. degree in biomedical engineering from Paris XII University, France, and the Postgraduate Diploma degree in biomedical engineering from the University of Technology of Compiègne, France. $\mathrm{He}$ is currently an Associate Professor with the Computer Science Department, Faculty of Science, University of Sfax. His research interest includes decision support system based on a knowledge discovery from data process. Most of his research are designed and evaluated in the medical field. He received the University Accreditation for his habilitation research in computer system engineering from the Engineering School of Sfax.

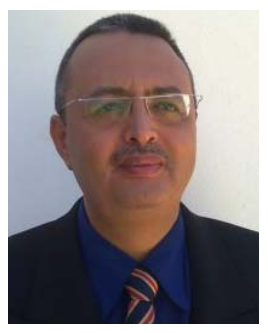

Adel M. Alimi (Senior Member, IEEE) graduated in electrical engineering in 1990. He received the $\mathrm{Ph} . \mathrm{D}$. and HDR degrees in electrical and computer engineering in 1995 and 2000, respectively. He has been a Full Professor in electrical engineering at ENIS, University of Sfax, since 2006, where he is currently the Founder and the Director of the REGIM-Laboratory on Intelligent Machines. His research interests include applications of intelligent methods (neural networks, fuzzy logic, and evolutionary algorithms) to pattern recognition, robotic systems, vision systems, and industrial processes. He focuses his research on intelligent pattern recognition, learning, analysis, and intelligent control of large-scale complex systems. He served as an associate editor and a member of the Editorial Board of many international scientific journals. He was also a guest editor of several special issues of international journals. He has also been serving as an Expert Evaluator for the European Agency for Research since 2009 . 